\title{
Rainer Kuhlen
}

\section{Napsterisierung und Venterisierung Bausteine zu einer politischen Ökonomie des Wissens}

\section{Formen des Umgangs mit Wissen und Information}

Aufgeschreckt durch spektakuläre Vorgänge wie die Napsterisierung von Wissen einerseits bzw. die Venterisierung von Wissen andererseits rückt die Frage, wie in elektronischen Räumen mit Wissen und Information umzugehen sei, ins Zentrum der Öffentlichkeit. Die Diskussion dieser Frage soll Bausteine einer künftigen politischen Ökonomie des Wissens unter den Bedingungen seiner Repräsentation in elektronischen Informationsprodukten und ihrer Verteilung in globalen Netzwerken liefern.

Aus der Perspektive der Informationswirtschaft bzw. der Wissensverwertungsindustrie ${ }^{1}$ wird mit „Napsterisierung“ die Aneignung und Weitergabe von Wissensprodukten unter Umgehung kommerzieller Gepflogenheiten des Handels mit diesen Produkten bezeichnet. „Napsterisierung“, quasi-synonym mit „Piraterie“, wird als polemischer bzw. kritischer Begriff verwendet, der impliziert, dass das, was durch Napsterisierung geschieht, als Verstoß gegen bestehende Gesetze oder sogar als Verstoß gegen ethische, in der bürgerlichen Gesellschaft allgemein anerkannte Prinzipien zu werten und entsprechend zu verfolgen sei. Um es in ein Schwarz-Weiß-Bild zu bringen: Mit der Bezeichnung „Napsterisierung“ wehrt sich die „alte“, nach kapitalistischmarktwirtschaftlichen Prinzipien organisierte Wirtschaft", einschließlich der „neuen“ Wirtschaft, die die Verwertungsformen der alten Wirtschaft im neuen Medium fortschreibt, gegen die „Hacker“-Welt in den elektronischen Räu-

1 „Informationswirtschaft“ und „Wissensverwertungsindustrie“ können synonym verwendet werden. In Abschnitt 2 versuchen wir den Zusammenhang von Wissen und Information und daraus abgeleiteten Begriffen für die Zwecke dieses Beitrags differenzierter herauszuarbeiten. Vorab: Informationswirtschaft erzielt einen immer größeren Anteil an der Erwirtschaftung des Bruttosozialprodukts dadurch, dass vorhandenes Wissen bzw. schon vorhandene Darstellungen von Wissen zu elektronischen Informationsprodukten aufbereitet und auf elektronischen Märkten gehandelt werden. Aktuelle Beispiele für Informationsprodukte: Online-Datenbanken, eBooks, digitalisierte Musik oder Videos als CD-ROM bzw. DVD als Online-Files; typische Vertreter der Informationswirtschaft: Text-, Bild-, Musik-, FilmVerlage, Datenbankanbieter, Betreiber elektronischer Marktplätze, Content Provider, ... 
men, nach deren Prinzipien und Praktiken Wissen vergesellschaftet und offen für jeden zugänglich gehalten werden soll. ${ }^{2}$

„Napster“ bzw. „Napsterisierung“, anfangs beschränkt auf den freien Austausch von digitaler Musik, bringe das auf den Punkt, was in elektronischen Räumen bei allen digitalen Wissensprodukten drohe - nämlich der Verlust an Kontrolle über die kommerzielle Verwertung von Wissen. Das Interesse der Informationswirtschaft, einschließlich das der Kulturindustrie - schon jetzt dominierende Bereiche der Wirtschaft insgesamt - stehe auf dem Spiel, wenn Napsterisierung zum allgemeinen Prinzip des Umgangs mit Wissen und Information werde. Gekoppelt - und damit moralisch mit mehr Aussicht auf allgemeine Zustimmung versehen - wird dabei das Interesse der Wissensverwertungsindustrie mit dem Interesse der Wissensproduzenten (Autoren, Künstler, Wissenschaftler), deren Anspruch an ihrem geistigem Eigentum, faktisch zwar in der Regel an die Verwerter abgetreten, durch Napsterisierung bedroht sei. ${ }^{3}$ Napsterisierung löse in der Missachtung des persönlichen geistigen Eigentums den Autorenbegriff auf und rüttele damit an den Grundfesten der abendländischen Kultur - so dramatisch durchaus Formulierungen aus der Informationswirtschaft.

Diejenigen, die von der Informationswirtschaft als „Napsterisierer“ gebrandmarkt werden, würden ihr Verhalten selber natürlich nicht als Napsterisierung bezeichnen, bzw. würden bei denjenigen, die bewusst danach handeln und nicht nur ihren individuellen Vorteil darin sehen, nicht akzeptieren, dass sie gegen ethische Prinzipien verstoßen. Vielmehr reklamieren sie, dass in elekt-

2 In Ermangelung einer besseren Bezeichnung verwenden wir hier den Ausdruck „HackerWelt”, so wie er in (Himanen 2001) benutzt wird. Himanen stellt der Max-Weber'schen Protestantischen Ethik als Ausdruck des Geistes des Kapitalismus die Hacker-Ethik gegenüber. In der Hacker-Ethik wird Information-sharing und das Offenhalten des Zugriffs auf Informations- und Computerressourcen, welcher Art auch immer, als ethische Pflicht angesehen. Konstruktiv setzt sich das z.B. in der Open-source-code-Wirtschaft um, radikalisiert wird das z.B. in dem von Richard Stallman bzw. der Free Software Foundation vertretenen GNU-Projekt (www.gnu.org), nach dem jedwede Information prinzipiell frei sein soll bzw. jede proprietäre Kontrolle über Wissen und Information zu bekämpfen sei (,information is community property and all software source should be shared“) (vgl. The Jargon file, Version 4.3.1., 29.6.2001; Stichworte GNU und hacker ethic: http://tuxedo.org/ esr/jargon/

jargon.html\#hacker\%20ethic); vgl. das GNU-Manifest (http://www.gnu.org/gnu/manifesto. html) oder, grundlegender und immer wieder zitiert, die Barlow'sche Declaration of the

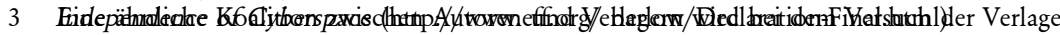
geschmiedet, die Reform des Urhebervertragsrechts, wie sie vom Bundesjustizministerium Mitte 2001 eingeleitet wurde (http://www.bmj.de/ggv/urhebver.pdf), zu verhindern. Die Hauptkritik richtet sich gegen die Verstärkung der Positionen der Autoren, z.B. durch Tarifverträge, mit denen Autoren sich besser gegen die dauerhafte Aneignung ihres Wissens gegenüber Verlagen schützen können. In ganzseitigen Anzeigen in den größeren Tageszeitungen (Mitte September 2001), unterzeichnet von den meisten deutschen Verlegern, heißt es: „Diese Reform ist rückschrittlich, weil sie das freie Verhältnis von Autor und Verlag aufhebt und es tariflich reglementieren will ... Das schadet nicht nur den Verlagen, es nützt auch den Urhebern nicht“ (z.B. Der Tagesspiegel, 14.9.2001, S. 33). 
ronischen Räumen neue Formen des Umgangs mit Wissen und damit auch neue ethische Prinzipien entstünden. Unethisch sei es eher, alte, in anderen medialen Kontexten entstandene Prinzipien und Formen der Sicherung des Rechts auf Eigentum an Wissen auf neue Kontexte, solche in elektronischen Räumen, zu übertragen. Schon gar nicht sei es mehr zu rechtfertigen, dass wenn alte Geschäftsmodelle mit hohen Transaktionskosten für Produktion und Verteilung von Informationsprodukten auf gegenwärtige, die Transaktionskosten gegen Null gehen lassende elektronische Räume übertragen würden.

Ist „Napsterisierung“ ein inzwischen zumindest im Angelsächsischen eingeführter Begriff, ${ }^{4}$ so prägen wir mit „Venterisierung“ eine neue Benennung. „Venterisierung“ im engeren, ursprünglichen Sinne bezieht sich auf die Ziele und Prozesse der von Craig Venter gegründeten Firma Celera Genomics, die sich die Entschlüsselung des Genoms von Lebewesen wie das der Fruchtfliege, der Maus oder des Menschen zum Ziel gesetzt hat, und zwar nicht, um interessenfreies Wissen zu erzeugen, sondern um das erarbeitete Wissen als Ware auf den Märkten, z.B. der Pharmaindustrie, die dieses Wissen in Produkte umsetzen kann, anzubieten und entsprechende Gewinne zu erzielen.

Venterisierung bedeutet Kommerzialisierung und Kontrolle von Wissen und Information. Der kommerzielle Aspekt ist natürlich kein neu entstandenes Phänomen. „Venterisierung“ benennt den weitergehenden perfektionierten Vorgang der kontrollierten, privaten Aneignung von Wissen (auch wenn dieses nicht mit privaten Mitteln unterstützt produziert wurde) und dessen Umsetzung in Informationsprodukte, die auf den Informationsmärkten im Sinne von kommerziellen Plattformen oder Marktplätzen gehandelt werden. Die gesamte neuzeitliche Entwicklung des Umgangs mit Wissen und Information kann als deren fortschreitende Kommerzialisierung gesehen werden (vgl. Kuhlen 1995). Die vorletzte Stufe in diesem Prozess hat die internationalen Online-Informationsmärkte der Fachinformation und -kommunikation entstehen lassen, mit ihren gut 10000 Datenbanken aus allen Wissensgebieten mit vielen Milliarden Einträgen (Fakten, Messdaten, bibliographische Angaben zu Publikationen, Volltexte, Statistiken, etc.), die, zunächst überwiegend mit öffentlichen Mitteln produziert und über entsprechende Informationseinrichtungen (Hosts) vertrieben, zunehmend in privater Trägerschaft geführt und nach Marktprinzipien gehandelt werden. Als vorläufig letzte Stufe hat sich die Informationswirtschaft der „neuen“ Online-Multi-/Hypermedia-Märkte im Umfeld des Internet etabliert.

Venterisierung, über das konkrete Beispiel Celera Genomics hinausgehend, radikalisiert die Kommerzialisierung von Wissen durch verschärfte Kontroll-

4 Eine Suche nach „Napsterization“ im internationalen Bereich von Google wies Ende August 2001 ca. 868 Treffer nach, zusätzlich 146 mit der englischen Schreibweise; 11 Treffer gab es zu „Napsterisierung“ aus Deutschland. 
mechanismen für digitale Wissensprodukte, durch die die bisherigen, die kommerzielle Nutzung einschränkenden Prinzipien des Fair use außer Kraft gesetzt werden können. ${ }^{5}$ Gemeint sind hier die Techniken des Digital Rights Management (DRM), durch die jede aktuelle Nutzung von Wissensobjekten in beliebig skalierbarer Größe genau registriert und abgerechnet bzw. genau festgelegt werden kann, in welchem Ausmaß die angebotenen Wissensobjekte bzw. Informationsprodukte überhaupt genutzt werden können. Fair use war der Kompromiss zwischen kommerzieller Verwertung und partieller Freizügigkeit der Nutzung im Interesse der Allgemeinheit, z.B. bei der Nutzung von Information durch die Wissenschaft oder der allgemeinen Öffentlichkeit in den öffentlichen und wissenschaftlichen Bibliotheken, aber auch der „Kompromiss“, dass ich das Buch, das ich gekauft und damit in Besitz genommen habe, an andere weitergeben darf, ohne dass diese dafür erneut bezahlen müssen. Fair use ist unter radikaler DRM-Anwendung nicht mehr gegeben. Die Einteilung in Wissenszonen, je nach Berechtigung oder Zahlungsbereitschaft bzw. -fähigkeit, die es in der Unterscheidung zwischen Informationsarmen und Informationsreichen natürlich immer schon gegeben hat und die die Grundlage der Verteilung von Macht in jeder Gesellschaft ist, werde, so die Kritik, durch DRM radikalisiert und stabilisiere den Status quo der aktuellen Besitz- und Machtverhältnisse.

„Venterisierung“ sollte, wenn er denn aufgenommen wird, ebenfalls als polemischer, kritischer Begriff verstanden werden. Er kritisiert, dass grundlegendes Wissen wie das über das Genom des Menschen nicht privat angeeignet werden darf, sondern, wie es durch das öffentlich finanzierte Human Genome Project (HUGO) geschieht, zur freien Nutzung aller uneingeschränkt öffentlich zugänglich gemacht werden muss. Der Venterisierung wird das Postulat „Wissen in den elektronischen Räumen will und muss frei sein“ entgegengesetzt. In elektronischen Räumen könne und müsse verwirklicht werden, was in der bürgerlichen Gesellschaft seit der Aufklärung wegen medialer Restriktionen nur annähernd zu erreichen war, nämlich Freiheit und Freizügigkeit beim Umgang mit Wissen und Information. Gekoppelt wird diese Kritik mit dem durch die allgemeine Architektur der Turing-Maschinen gestützten Handlungsoptimismus, dass faktisch jede Stufe der Venterisierung unterlaufen werden kann. Jede durch Software vollzogene Kontrolle über elektronisch dargestelltes und verteiltes Wissen könne immer schon durch andere Software außer Kraft gesetzt werden. ${ }^{6}$

5 Wir verwenden den aus dem amerikanischen Copyright stammenden Begriff des Fair use. In der deutschen Gesetzgebung entspricht das den „Schranken des Urheberrechts“ (§45-63 des Urheberrechtsgesetzes), die dem öffentlichen, aber auch berechtigten privaten Interesse an einer gewissen Freizügigkeit bei der Nutzung geistiger Produkte Rechnung tragen sollen. Wie wir sehen werden, geht der gegenwärtige Streit (zwischen Informationswirtschaft, Hacker-Welt und staatlichen Institutionen) in erster Linie darüber, inwieweit Software durch 
Die so kritisierten Wissensverwerter sehen sich natürlich nicht als „Venterisierer“. Vielmehr sehen sie es als selbstverständlich, sicher aber als berechtigt und konform mit den allgemeinen Prinzipien von Wirtschaft und Gesellschaft an, dass durch private Investitionen entstandenes Wissen bzw. durch informationelle Aufbereitungsarbeit entstandene Informationsprodukte allemal privates Eigentum seien und entsprechend auf den Märkten geschützt werden dürften. Verstöße gegen die aktuelle Venterisierungspraxis, wie sie aus der Hacker-Ethik heraus für legitim erklärt werden, seien nichts anderes als Napsterisierung in der Ausprägung als Piraterie.

Um es erneut in ein Schwarz-Weiß-Bild zu bringen: Mit der Bezeichnung „Venterisierung“ greift die nach Hacker-Ethik-Prinzipien organisierte und fundierte Basisbewegung der Informationsgesellschaft die „alte“ und „neue“, nach kapitalistisch-marktwirtschaftlichen Prinzipien organisierte Informationswirtschaft an, die, ähnlich konsequent, aber eben ganz anders als in der Hacker-Ethik, in den elektronischen Räumen die Vollendung der in der kapitalistischen Warenwirtschaft angelegten Verwertung bzw. der privaten Aneignung von Wissen bzw. der Kontrolle über dessen Verwertung sieht.

Natürlich sind diese verschiedenen Ausprägungen einer politischen Ökonomie von Wissen schwarz-weiß gezeichnet. Weder gibt es die „reine“ bzw. aus der anderen Sicht die „böse“ Hacker-Informationswelt noch gibt es die konsequent verwertende, jede öffentliche Nutzung reglementierende und jeden Fair use ausschließende Informationswirtschaft. Wir werden im Folgenden versuchen, die in den beiden Konzepten der Napsterisierung und Venterisierung angelegten polemischen Implikationen zu vermeiden und auf Wechselwirkungen zwischen diesen beiden aktuellen Ausprägungen einer politische Ökonomie des Wissen hinweisen. Dazu fassen wir noch einmal die beiden wesentlichen Punkte der Napsterisierung zusammen:

„Napsterisierung“ benennt a) den Vorgang der weitgehend unbehinderten persönlichen Aneignung und Verteilung von repräsentierten Wissen auf Informationsmärkten im Sinne von öffentlichen Foren oder öffentlichen Netzwerken $^{7}$ - in diesem Sinne kann man auch von Vergesellschaftung von Wissen sprechen. Napsterisierung bedeutet aber auch - wie wir näher herausarbeiten

das Recht auf freie Meinungsäußerung geschützt und damit frei verteilt werden dürfe (also auch Software, die bestehende Software außer Kraft setzt) oder ob solche Software prinzipiell verboten werden kann, wie es in den USA durch das DMCA (vgl. Anm. 16) geschehen ist. Aber auch jede staatliche Reglementierung kann faktisch durch Softwarekonstellationen unterlaufen werden, indem z.B. Dekodierungssoftware dergestalt modularisiert verteilt wird, dass jedes Teil für sich unproblematisch ist und erst durch das Zusammenfügen wirksam wird.

7 In Kuhlen (1995) haben wir auf dieses doppelte Verständnis von Informationsmärkten als öffentliche Foren des freien Austauschs von Wissen und als kommerzielle Marktplätze des Handels mit Informationsprodukten hingewiesen und die Chancen und Risiken einer Konkurrenz bzw. Kooperation zwischen beiden Märkten diskutiert; vgl. auch Kuhlen (1998). 
wollen - b) ein der globalen Netzwelt angemessenes neues Organisationsmodell (und dann auch Geschäftsmodell) für die Produktion und Nutzung von Wissen, das auf den Prinzipien des Information sharing und der verteilten Eingabe von Wissensobjekten und ihres Abrufs beruht. Napsterisierung in diesem Sinne ist also ein Gegenmodell zur zentralen Organisation und Verteilung von Wissen, oder allgemeiner formuliert: Napsterisierung ist eine Reaktion auf die in einem aktuellen Zustand der Venterisierung auftretenden Widersprüche zwischen Produktions- und Verteilungsformen einerseits und der durch neue mediale Umgebungen möglichen Potenziale.

In der Regel geht bislang die Venterisierung von Wissen mit einer Zentralisierung der Organisation und Verteilung von Wissen zusammen, da im kommerziellen Geschäftsmodell die Kontrolle über die Vorgänge eher zentral organisiert ist. Das muss natürlich nicht so bleiben. Das Gegenteil ist zu erwarten. Wie das Open-Source-Modell der Softwareerstellung zeigt, finden alternative Konzepte - alternative Produktions-, Verteil- und Nutzungsformen, kurz: offene und in der Regel auch dezentrale Organisationsformen - Eingang in die kommerzielle Verwertung ${ }^{8}$. Man kann diesen Gedanken vermutlich verallgemeinern: die Napsterisierung von Wissen scheint die Voraussetzung für die nachfolgende Venterisierung von Wissen zu sein, unter der Bedingung, dass durch diese neuen Organisationsformen bisherige Transaktionskosten verringert oder ganz beseitigt und damit Gewinnerwartungen gesteigert werden können.

$\mathrm{Zu}$ jedem historischen Zeitpunkt wenden sich die Status-quo-Venterisierer gegen die Napsterisierung, gegen das Unterlaufen des Status quo am Besitz von Wissen und an den Verteilmechanismen - in der Regel allerdings kaum lange erfolgreich, wenn man als Kriterium für Erfolg die Marktdominanz ansieht. Aus jedem Zustand der Napsterisierung entstehen in der kapitalistischen Verwertungsgesellschaft neue Geschäftsmodelle und damit neue Formen der Venterisierung von Wissen. Die Wirtschaft erteilt der „Piraterie“ quasi Absoluti-

8 Z.B. bei der Apache-Server-Software bzw. der Offenlegung des Solaris-Code, auch, wenn auch verzögert, bei der Offenlegung des Codes der Netscape-Communicator-BrowserSoftware; ähnliche, in der Regel auf LINUX bezogene, wenn auch kontrollierter auf die eigenen Interessen ausgerichtete Entwicklungen bei Dell, Hewlett Packard (http://www.hp.com/hpinfo/newsroom/feature_stories/opensource01.htm), Intel und IBM vgl. auch den Einsatz von Open-Source-Software (OSS) im Verlagshaus O'Reilly (http://opensource.oreilly.com/; vgl. http://conferences.oreilly.com/oscon/tim_letter.html „open source has grown from the hacker's secret weapon to a major force in serious enterprise computing"). Das folgende Zitat (aus einer internen Microsoft-Stellungnahme zu OSS) belegt exemplarisch, dass OSS von traditionellen Softwareformen wie Microsoft als Bedrohung empfunden wurde: „OSS poses a direct, short-term revenue and platform threat to Microsoft, particularly in server space. Additionally, the intrinsic parallelism and free idea exchange in OSS has benefits that are not replicable with our current licensing model and therefore present a long term developer mindshare threat" (http://www.extropia.com/press/ microsoft_on_open_source.html). Ähnliches gilt für das allgemeine Napsterisierungsmodell. 
on. Die Störtebeckers und Robin Hoods werden zu CEOs. Cisco, Dell, Sun, Apple (und viele andere) sind schon klassische Beispiele für die Entwicklung neuer Geschäftsmodelle, hier auf der Grundlage des Open-Source-Gedankens (vgl. Anm. 8). Napsterisierung (jetzt verstanden als die Entwicklung neuer freier Formen des Umgangs mit Wissen und Information) scheint in der Informationsgesellschaft die Bedingung für Inventionen auf den Gebieten der Informations- und Kommunikationstechnologien, sicherlich auch der GenTechnologie, zu sein, wenn auch auf Dauer - so formuliert es Castells - „financing, manufacturing, and marketing will ultimately decide which technologies survive in the marketplace" (Castells 2001, 177). Ist Napsterisierung die Bedingung für Invention, so scheint - bislang - Venterisierung die Bedingung für Innovationen zu sein (wenn man im Einklang mit den Wirtschaftswissenschaften unter „Innovationen“ marktfähig umgesetzte und kommerziell genutzte Produkte oder Dienstleistungen versteht bzw. neue, die bisherige kommerzielle Praxis zur Erstellung und Verteilung von Wissens- und Informationsprodukten verbessernde Organisationsformen ${ }^{9}$ ). Ob dies ein Grundgesetz einer neuen politischen Ökonomie ist, ist aus den bisherigen empirischen Hinweisen nicht zureichend abzuleiten - zumindest die faktischen „Napsterisierer" würden dies bestreiten und Venterisierung als Relikt einer veralteten Gesellschafts- und Wirtschaftsform ansehen, aus der innovative Modelle prinzipiell nicht mehr entstehen können.

\section{Wissen und Information}

Wir haben bislang „Wissen“ und „Information“ bzw. Wissens- und Informationsprodukte weitgehend austauschbar verwendet. Das ist auch häufig in der Literatur der Fall. Unsicher, wie man beide Begriffe voneinander absetzen soll, werden in der Regel beide zusammen verwendet, in der Hoffnung, dass der jeweilige Leser schon wissen wird, was gemeint ist. Zur Beantwortung oder bescheidener: zur Diskussion der Frage nach dem Besitz oder der Verfügung über Wissen und Information ist es jedoch wichtig, Unterscheidungen vorzunehmen. Das soll in diesem Abschnitt geschehen.

Dass Wissen und Information Schlüsselfaktoren für die Entwicklung der gesellschaftlichen Großbereiche - Wirtschaft, Politik, Verwaltung, Wissenschaft,

9 „Innovation“ wird in der Wirtschaftswissenschaft über „marktfähig“ und „kommerziell“ definiert. Die kommerzielle Ausrichtung muss aber nicht für Innovation zwingend sein. Insofern könnte und sollte der Innovationsbegriff durchaus auch für „Produkte“ aus der OpenSource-Umgebung bzw. freie Angebote jeder Art verwendet werden, insofern sie eben auf den Informationsmärkten (frei) verfügbar sind. Dies gilt insbesondere auch für neue Organisationsformen in der Distribution und Nutzung von solchen Produkten, wie sie in den P2P-Modellen immer größere Verbreitung finden (vgl. Anm. 29). Bei dieser Sicht würde man bei der Übernahme von innovativen Modellen aus der Open-Source- bzw. Information-Sharing-Umgebung in die herkömmliche Informationswirtschaft von „Diffusion“ sprechen. 
Ausbildung und Medien - bzw. für die in ihnen handelnden Organisationen und Individuen sind, bedarf heute keiner weiteren Begründung. $\mathrm{Zu}$ sehr sind die entsprechenden Begriffe wie „Informationsgesellschaft“ oder „Wissensgesellschaft“ Bestandteile des Alltagsvokabulars und des öffentlichen Bewusstseins geworden. ${ }^{10}$ Mit diesen Begriffen werden im allgemeinen Aussagen verbunden wie, (i) dass über Wissen und Information bzw. über Informationsund Wissensprodukte und -dienstleistungen der größte Teil des Bruttosozialprodukts erwirtschaftet und der größte Teil der Arbeitsplätze bereitgestellt wird und (ii) dass im Prinzip alle Lebensbereiche und in ihnen alle Menschen von der fortschreitenden Informatisierung betroffen sind, d.h. dass überall Informations- und Kommunikationsdienste und die zu ihrer Verfügbarmachung notwendigen Technologien zum Einsatz kommen. Diese Dienste werden in den Konsequenzen, je nach gesellschaftspolitischer Position, durchaus uneinheitlich, positiv/optimistisch oder negativ/pessimistisch eingeschätzt, vor allem auch durchaus unterschiedlich bezüglich der Einschätzung, welche Interessen an Information und Wissen Chancen haben sich durchzusetzen. Abweichend von bzw. ergänzend zu der unverkennbaren Dominanz wirtschaftswissenschaftlicher, informationstechnischer und sozialwissenschaftlicher bzw. sozialpsychologischer Sichten auf Informationsgesellschaft und Wissensgesellschaft steht im Folgenden eine informationswissenschaftliche Perspektive im Vordergrund. Danach sollten Gesellschaften nur dann zu Recht als Informations- und Wissensgesellschaften bezeichnet werden, wenn sie ihren Mitgliedern die Möglichkeit eröffnen, ihre privaten, professionellen und öffentlichen „Geschäfte“ auf informationell abgesicherter Grundlage zu fairen, akzeptablen Bedingungen durchzuführen. Informationswissenschaftliche Verfahren sollen in dieser emanzipativen Ausrichtung Informations(nutzungs)barrieren aufdecken bzw. dazu beitragen, diese Barrieren abzubauen.

Als experimentell konstruktive Disziplin hat die Informationswissenschaft die Entwicklung von Verfahren und Systemen zum Gegenstand, durch die vorhandenes Wissen so gesammelt, aufbereitet und zugänglich gemacht wird, dass es als Information in konkreten Handlungszusammenhängen genutzt werden kann. Damit sind wir bei dem Unterschied von Wissen und Information. ${ }^{11}$

10 (Castells 2001) weist allerdings zu Recht darauf hin, dass Wissen und Information (auch er benutzt beide Begriffe parallel und damit synonym) zentral für alle Gesellschaften waren: „all economies are knowledge-based economies and all societies are, at their core, information societies” (159). Er vermeidet daher Begriffe wie „Wissenswirtschaft“ oder „Informationsgesellschaft“ und spricht bevorzugt von „Informationalism“ als das neue durch die gegenwärtige Informations- und Kommunikationstechnologie möglich gewordene Paradigma der Erweiterung der menschlichen Informationsverarbeitungskapazität.

11 Fast überflüssig zu betonen, dass, angesichts der Vielschichtigkeit von Wissen und Information, selbst in der engeren Fachwelt der Informationswissenschaft keineswegs Einigkeit über die hier vorgetragenen Begriffsbestimmungen von Wissen und Information besteht. Wir verfolgen hier die pragmatische Konzeption von Information, weil sie uns für die Erklärung des Umgangs mit Wissen und der Prozesse auf den Märkten des „Handels“ mit Wissen 
Wissen ist ein auf kognitive Strukturen bezogenes Konzept. Wissen ist die zu einem bestimmten Zeitpunkt durch Anwendung bestimmter Verfahren (Erfahrung, Experimente, Logik, Kommunikation) gewonnene und als gültig angesehene Menge der Aussagen über Objekte und Sachverhalte der Welt, wobei das Ausmaß der reklamierten Gültigkeit in der Bandbreite von „Wahrheit" (gültig nach dem Stand der Wissenschaft) bis hin zur offensichtlichen Lüge bestehen kann. Information hingegen steht orthogonal dazu und sagt entsprechend nichts über den Wahrheitswert des zugrundeliegenden Wissens aus. Information wird aus bestehendem Wissen erarbeitet.

Wissen wird zur Information nur durch den Bezug auf einen aktuellen Handlungszusammenhang (Information ist Wissen in Aktion). Dies macht den pragmatischen Charakter von Information aus, während Wissen nicht notwendigerweise auf einen bestimmten Nutzungskontext bezogen ist. Nach dieser Unterscheidung macht die Frage nach dem Besitz von Wissen, wird sie denn wörtlich genommen, gar keinen Sinn. ${ }^{12}$ Wissen als interne kognitive Struktur ist für sich nicht einsehbar und damit nicht Gegenstand kommerzieller Aneignung, jedenfalls solange die Wissenschaft noch nicht in der Lage ist, Wissensstrukturen in menschlichen Gehirnen über die bloße Lokalisierung hinaus zugänglich, d.h. „lesbar" zu machen. ${ }^{13}$ Wenn menschliches Wissen (noch) nicht von Gehirn zu Gehirn direkt „heruntergeladen“ werden kann, muss Wissen in irgendeiner Form repräsentiert und als Information zugänglich gemacht werden. Erst mit der Repräsentation von Wissen in reproduzierbaren Zeichen- und Codierungssystemen, der Abspeicherung in rezipierbaren Speichern und den Möglichkeiten der selektiven Verteilung beginnt die Aus-

mächtiger zu sein scheint, als z.B. eine informations-/nachrichtentheoretische Sicht, die die quantitativen, messbaren, syntaktischen Eigenschaften von Information betont und dabei den Kontext und die Rahmenbedingungen der realen Nutzung außen vor lässt. Eine informationstheoretische Aussage, wie z.B. dass ein Wissensobjekt eine bestimmte Anzahl an in Bits messbaren Informationen enthält, kann durchaus sinnvoll sein, aus unserer Sicht ist nur das aus dem Wissensobjekt eine Information für einen Benutzer, was er noch nicht kennt und was er aktuell tatsächlich benötigt.

12 Vgl. allerdings die beiden jüngsten Konferenzen der Heinrich-Böll-Stiftung, die sich mit der Frage nach dem Besitz von Wissen auseinandergesetzt haben (http://www.boell.de/ index01.htm); vgl. dazu die beiden einleitenden Vorträge (Kuhlen 2000) und (Kuhlen 2001).

13 Klassisch ist hierfür der Text von (Moravec 1988), der in seiner wilden Utopie bzw. Dystopie der sukzessiven Substitution des Menschen durch künstliche Maschinen immer wieder als unseriös und als Science fiction von der Fachwelt zurückgewiesen wurde. Der Gedanke, einmal in die Welt gesetzt, ist geblieben; vgl. das Interview mit Ray Kurzweil (http://www.unesco.org/courier/2001_07/uk/doss42.htm\#top): „By 2030, we will be able to send billions of nanobots inside the human brain to communicate wirelessly with billions of different points in the brain. They will give us the ability to combine our biological intelligence with non-biological intelligence. Ultimately you will have entities that are completely non-biological, with copies of human brains derived from reverse engineering, and you will have biological humans that have billions or trillions of nanobots in their brains augmenting their intelligence or enabling them to exist in virtual reality. So we will no longer be able to say humans on the left, machines on the right" (vgl. Kurzweil 1999). 
einandersetzung um die Verfügung über Wissen.

Die Repräsentation und damit die Reproduktion von Wissen stellen sozusagen den Sündenfall des Umgangs mit Wissen dar. Die Repräsentationen von Wissen können sich Dritte aneignen, und diese können damit beginnen, den Austausch von Wissen, jenseits von Angesicht zu Angesicht (F2F), zu organisieren und Handel damit zu betreiben, ohne dass der originale Wissensproduzent davon wissen muss geschweige denn - wie es ja auch in der längsten Zeit der Menschheitsgeschichte der Fall war - dafür belohnt werden müsste. Zwischen Wissensproduzent und Nutzer treten die Wissensrepräsentation und damit die Formen der Vermittlung über Informationsprodukte.

Die Umsetzung von Wissen in Wissen repräsentierende Informationsprodukte, einschließlich ihrer Verteilung und Vermittlung, sind im Verlauf der Geschichte immer ressourcenaufwendiger geworden. Die Transformation von Wissen geschieht nicht von selbst, sondern wird durch Arbeit, Informationsarbeit, erreicht. Informationsarbeit hämmert Keilschrift in den Stein, lässt Pergament- und Papierhandschriften entstehen, schafft Maschinen und Verfahren für den Druck mit bewegten Lettern, erstellt Inhaltsverzeichnisse, Register, Abstracts, Übersetzungen von Texten, produziert Datenbanken und schafft digitale Repräsentationen von multimedialen Objekten, die hypertextuell verknüpft elektronische Netzwelten erzeugen. ${ }^{14}$

Informationsarbeit, zunehmend differenzierter geworden, erzeugt fortlaufend informationelle Mehrwerte gegenüber dem einfacheren ursprünglichen Repräsentationsobjekt, z.B. die Datenbank- oder Hypertextversion gegenüber dem ursprünglichen linearen Text. In die Erzeugung dieser Mehrwerte und die Formen der Verteilung der daraus entstehenden Produkte muss investiert werden. Diese Investitionen werden spätestens mit den Organisationsformen der modernen bürgerlichen Gesellschaft überwiegend mit privatem Kapital getätigt und werden mit einer entsprechenden Gewinnerwartung wie auch bei anderen Produkten beliebiger Art unternommen. Entsprechend scheint es selbstverständlich zu sein, dass für Wissen repräsentierende Informationsprodukte mit Mehrwerteigenschaften Entgelte entrichtet werden müssen, und zwar weniger an die originalen Wissensproduzenten als an die Wissensverwer-

14 Schon diese fast willkürliche Auflistung der Entwicklung von Repräsentationsformen und dadurch möglich werdenden Informationsprodukten zeigt, dass die Darstellung von Wissen und seine Verteilung und Nutzung über im Printmedium realisierte Zeichen nur eine bestimmte Epoche in der Geschichte der Wissensvermittlung darstellt. Damit ist nicht ausgesagt, dass diese Epoche durch andere Formen endgültig abgelöst wird (schon gar nicht, dass dieses Ende heute unmittelbar bevorsteht) - Printmedien oder andere materialisierte elektronische Produkte wie die gegenwärtige CD-ROM als bislang verbreitetster Träger von multimedialer Information (Musik) oder DVD für bewegte Bilder müssen nicht vollständig verschwinden. Wahrscheinlich aber ist, dass frühe mediale Repäsentations- bzw. Speicherformen, wie Schallplatte, Tonband, klassische Filme, Video, nicht-digitale Fotografie, nicht mehr die den „Fortschritt“ der Nutzung bestimmenden Medien sein werden. 
ter (Verlage, Content Provider). Dieser Refinanzierungs- und Gewinnanspruch an Informationsprodukte bewirkt eine Verknappung des an sich im Überfluss vorhandenen Gutes „Wissen“ und teilt Gesellschaften in Wissenszonen ein, für diejenigen, die diesem Anspruch entsprechen können und wollen (Information rich) und diejenigen, die das nicht können (Information poor).

Trotz dieser Verknappung ist die bisherige Geschichte der Nutzung von Wissen eine mehr oder weniger gradlinige Entwicklung von den Wissensmonopolen einiger zu den Wissensegalitäten im Prinzip aller gewesen. Die mediale Entwicklung mit den Möglichkeiten immer größere Nutzungsschichten einschließender Repräsentations- und Verteilformen kompensiert (bislang) die systematische Verknappung der kommerziellen Verwertung von Wissen. Man kann es natürlich auch so formulieren, dass erst die Bereitschaft, umfassend in die Erzeugung informationeller Mehrwerte $\mathrm{zu}$ investieren und entsprechende Technologie für Produktion und Verteilung von Informationsprodukten zu erzeugen, die Masseninformationsmärkte hat entstehen lassen, die Informationsprodukte für alle erschwinglich machen. Der Beweis für globale Märkte, nämlich das bestehende Digital divide zu überwinden, muss allerdings erst noch erbracht werden.

Konflikte in neuen Phasen der Wissensrepräsentation und der Erzeugung von Informationsprodukten entstehen vor allem dann, wenn alte Organisationsund Verteilungsmodelle und daraus abgeleitete Besitzansprüche auf die neue Medienumwelt übertragen werden ${ }^{15}$. Das ist weitgehend der Ursprung des gegenwärtigen Konfliktes und der Auseinandersetzung um Napsterisierung und Venterisierung von Wissen - drastisch an dem originalen Napster-Konflikt ersichtlich, der nicht zuletzt dadurch entstanden ist, dass Verwertungsansprüche an Informationsprodukten wie Schallplatten und auch noch an CD-ROMs auf digitale Netzprodukte mit Transaktionskosten, die gegen Null gehen, übertragen wurden. Der Vorgang der umfassenderen Napsterisierung spiegelt diesen Konflikt wider.

Der Protest der Netzwelt gegenüber der klassischen Wissensverwertungsindustrie, diese bislang weitgehend unterstützt von den staatlichen Institutionen durch die von ihnen getroffenen rechtlichen Regelungen ${ }^{16}$, hat sich in den

15 (Deibert 1997) hat wie viele andere Autoren aus dem Umfeld der Medientheorie aufgezeigt, wie die Entwicklung des Buchdrucks mit bewegten Lettern die Produktions-, Verteil- und Nutzungsbedingungen für Handschriften obsolet gemacht hat und damit auch die Herrschaftsansprüche derjenigen (in diesem Fall in erster Linie die Hierarchien der katholischen Kirche), die das Monopol an dem in diesen alten medialen Formen repräsentierten Wissen gehabt haben. Entsprechend erfolgreich war die neue protestantische Bewegung, die sich die Vorteile der neuen Wissenstechnologie schneller aneignen konnte als das alte System. Natürlich sind der Protestantismus und die moderne bürgerliche Gesellschaft nicht durch den Druck mit bewegten Lettern entstanden, aber sicherlich die Entwicklung neuer Produktions- und Verteilformen allgemein durch die Verwertung der Potenziale der neuen Technologie.

16 Exemplarisch hierfür das 1998 vom US-Kongress verabschiedete „Digital Millennium Co- 
letzten Jahren immer stärker artikuliert, und zwar sowohl von Seiten der Wissenschaft als auch von Seiten der „Hacker-Welt“. Der Wissenschaft, sowohl den Autoren selber als auch den Wissenschaftsverbänden und Bibliotheken/Rechenzentren, stehen dafür inzwischen mächtige Formen der Selbst- und Direktpublikation bzw. der Vermittlung zur Verfügung. Aus der Hacker-Welt wird in erster Linie Software entwickelt, z.B. die Dekodierung verschlüsselter Produkte, um die Reichweite dessen, was Lawrence Lessig „Zoning“ genannt hatte, einzuschränken oder zu unterlaufen. ${ }^{17}$ Der Protest artikuliert sich aber durch das Marktverhalten der Endnutzer selber, die durch neue Organisations- und Verteilungsmodelle, aber auch durch frei verfügbare Software, in die Lage versetzt werden, legale Verwertungsansprüche der Wissensindustrie zu unterlaufen - im unproblematischen Fall durch Verzicht auf die kommerzielle Software, wenn vergleichbare freie Software vorhanden ist ${ }^{18}$, im problematischen Fall durch Ignorieren bestehender Rechtsvorschriften. Und sie tun das auch in großem Stil, nicht zuletzt auch deshalb, weil die Preispolitik für digitale Produkte (CD-ROM, DVD), die sich eher an den Preisen für Produkte in anderen Medien mit ganz anderen Transaktionskosten orientiert, nicht als

pyright Act“ (DMCA), durch das die Rolle der Wissensverwertungsindustrie mit Blick auf elektronische Produkte verstärkt wurde. In der EU wird versucht, über eine entsprechende Richtlinie zum Urheberrecht in der Informationsgesellschaft eine Harmonisierung des EURechts beim Urheberrecht zu erreichen, die im Einklang mit dem Abkommen der World Intellectual Property Organisation (WIPO) von 1996 und dem DMCA ist. Wie in der EU üblich, müssen die Mitgliedstaaten nationale Gesetzesanpassungen innerhalb von 18 Monaten vornehmen, die ihnen allerdings Spielräume, z.B. zwischen durchgängigem DRM oder Pauschalabgaben über Hardware, offen lassen.

17 (Lessig 1999) wies darauf hin, dass die Kommerzialisierung der Informationsmärkte, entgegen der ursprünglichen freien Wissensnutzung im Internet - eine fortschreitende Einteilung der Domänen des Wissens in Nutzungszonen zwingend erforderlich macht - z.B. Zonierung über Passwörter (einschließlich biologische Authentifizierungs/Identifizierungsverfahren), über entsprechende Lizenzen oder bekundete Zahlungsbereitschaft, aufgrund von Nutzungskompetenzen, nachgewiesenem Nutzungsinteresse oder kontrollierter politischer Zuverlässigkeit/Korrektheit - um nur einige Kriterien zu nennen. Technologische Basis hierfür sind, wie erwähnt, die immer weiter zum Einsatz kommenden Softwareprodukte des Digital rights management, die dadurch ein leichteres Spiel bekommen, das über die Verwirklichung des Konzepts des Digital object jedes noch so kleine Stück Wissen referenzierbar, nachspürbar, abrechenbar und damit kontrollierbar wird (vgl. http://www.doi.org/); vgl. den W3C DRM Workshop 2001 (http://www.w3.org/2000/12/drm-ws/pp/Overview.html).

18 Einschlägig hier das Marktverhalten der Endnutzer bei der Verwendung von StarOffice (vergleichbar in der Leistung mit dem kommerziellen Microsoft-Office-Paket). Bis Mitte 2001 wurde 5 Millionen Mal StarOffice online heruntergeladen. Insgesamt wurden bis dahin 20 Millionen kostenlose Lizenzen erteilt (http://www.sun.com/software/star/staroffice/5.2/ news/fivemillion.html). Immer populärer wird die Nutzung dieser (und vergleichbarer) Software auch bei öffentlich finanzierten Einrichtungen. Ein Beispiel: Die Polizei in Schottland mit 1000 Benutzern hat Mitte 2001 vollständig auf StarOffice umgestellt und hat dadurch etwa eine Viertel Million Pfund eingespart (http://www.vnunet.com/Print/1124456). Auch in Ausbildungseinrichtungen jeder Art erweist sich dies zunehmend als Alternative zum kommerziellen teuren Angebot. 
nachvollziehbar und nicht als angemessen oder gerecht angesehen wird (vgl. Werner 2001; Manasse 2000). Dies hat zum einen einen fortschreitenden Vertrauens- und damit auch Loyalitätsverlust gegenüber den Anbietern von Wissensprodukten und ihren DRM-Techniken zur Folge ${ }^{19}$, und löst zum andern, mit dem Vertrauensverlust einhergehend, die Verbindlichkeit bestehender ethischer Prinzipien (Achtung und Schutz geistigen Eigentums) auf und machen das Durchsetzen bestehender rechtlicher Schutzbestimmungen fragwürdig. Faktisches Verhalten als Massenphänomen stellt bestehende Ethik und bestehendes Recht in Frage. Und es ist offen, ob Rechtsanpassungen oder DRM-Regelungen diese wieder rekonstituieren können.

\section{Napsterisierung}

Wir erinnern daran, dass wir den Ausdruck „Napsterisierung“ nicht nur auf die Vorgänge bezogen haben, die direkt mit der MP3-Tauschbörse Napster assoziiert werden, sondern ihn gleichsam als Metapher für eine neue, auf Teilen und verteilte Nutzung beruhende Organisationsform im Umgang mit Informationsprodukten vorgeschlagen haben. Um diesen Gedanken zu konkretisieren, konzentrieren wir uns im Folgenden auf vier Themen: der Streit um Napster und alternative P2P-Modelle, der Streit um Verschlüsselung und Dekodierung digitaler Objekte am Beispiel DVD bzw. deCSS und ebooks und Perspektiven der Napsterisierung in Ausbildung und Wissenschaft.

\section{1 Napster}

Napsterisierung wird in erster Linie mit Wissensobjekten in Form von digitalen Musiktiteln in Verbindung gebracht, die über das Netz bzw. über die Vermittlung durch die Napster-Börse (www.napster.com) getauscht werden. Napster beruht auf der Verbindung einer neuen Technologie (MP3) ${ }^{20}$ und einer neuen Organisationsidee, der Idee des Person-to-person-non-commercial

19 Vgl. zur Vertrauensproblematik von DRM-Software (Cheng/Litva/Main 2001)

20 MP3-Technologie (MPEG=Moving Picture Experts Gropu Audio Layer-3), entwickelt seit 1987 am Fraunhofer-Institut für Integrierte Schaltungen und 1993 und 1998 von der ISO zum Standard erklärt, ist im wesentlichen ein frei verfügbares Komprimierungsverfahren, das übertragungsintensive digitalisierte Musik dermaßen im Umfang reduziert (um etwa den Faktor 12), dass selbst bei Übertragungsraten, die über traditionelle Modems (z.B. 64 KB) erreicht werden, das Downloading zumindest kürzerer Musikstücke akzeptabel wird und ohne für das menschliche Ohr wahrnehmbare Qualitätsverluste. Mit der immer weiteren Verbreitung von ISDN oder DSL-Technologie auch in privaten Umgebungen rückt die Übertragung ganzer CD-ROMs in den Bereich des Zumutbaren. Da zur gleichen Zeit auch die Möglichkeit des individuellen „Brennens“ von CD-ROMs für Endnutzer zu akzeptablen Kosten gegeben war, setzte dies Nutzer in die Lage, die nach dem Dekodieren speicherintensive Musik auf externe Dateien auszulagern und damit für die Nutzung in computerunabhängigen Wiedergabegeräten bereitzuhalten. 
file sharing ${ }^{21}$. Napster hatte als selbständige Organisation keineswegs den kommerziellen Handel mit Musikprodukten intendiert oder ermutigt, sondern eher ein kommunikatives Ziel verfolgt - in der Formulierung der früheren CEO Eileen Richardson: „What we want is to help people find music and build community around genres of music" (http://www.mp3.com/news/ 436.html) - sicherlich auch in der Erwartung, dass bei intensiver Nutzung des Napster-Softwareangebots attraktive Werbeeinnahmen erzielt werden können, und sicherlich auch in der Fehleinschätzung, dass die Wissensindustrie die aus ihrer Sicht fortlaufende faktische Copyright-Verletzung einfach hinnehmen würde.

Der Erfolg gab Napster und dem Organisationsmodell Recht - bevor Napster durch gerichtliche Intervention auf Grund der Klage von RIAA (The Recording Industry Association of America ${ }^{22}$ in seinen Aktivitäten und damit in seinem Nutzungsangebot eingeschränkt wurde, waren gut 30 Millionen Nutzer bei Napster registriert. Selbst im August 2001, an einem beliebigen Vormittag waren noch gut 70.000 „Napsterisierer“ online.

Das Napster-Geschäftsmodell für den Umgang mit Wissen, am Beispiel von Musikobjekten, war für jedermann transparent und in der Kombination der Komponenten offenbar für sehr viele Musikliebhaber attraktiv. Napster brachte die Funktionen Kommunikation (über ein Chat-Angebot, in dessen Chat-Rooms über Musik diskutiert werden kann), Information (Hintergrundinformation zu Musikstücken, Interpreten, Gruppen etc.) und vor allem Transaktion nach dem Prinzip des Information sharing. zusammen. Napster stellt(e) keine Musikobjekte bereit, sondern die Software, mit der digitalisierte Musik von jedem registrierten Benutzer in Verzeichnissen seines eigenen Rechners zum Herunterladen bereitgehalten wurde. Gesucht und gefunden werden können Musikobjekte natürlich nur dann, wenn die „Anbieter“ online sind. Ist ein Musikstück mit einer Quelle identifiziert, erlaubt die NapsterSoftware den Zugriff zu den das gewünschte Musikstück enthaltenen Verzeichnissen, die von den Anbietern als „offen“ deklariert worden sind ${ }^{23}$. Das Sharing-Modell macht, vielleicht naiv, das im globalen Maßstab möglich, was im privaten Bereich immer der Fall und erlaubt war: die Weitergabe von ein-

21 http://www.napster.com/pressroom/qanda.html; interessanterweise hatten über ein Drittel der Napster-Nutzer bei einer Umfrage nach dem Grund für die Nutzung des Dienstes bzw. für dessen Rechtfertigung angegeben: „It's like the public library“ (http://www.idc.com/ ebusinesstrends/ebt20010830.htm).

22 RIAA vertrat die Interessen von EMI Recorded Music, Sony Music Group, Universal Music Group, Warner Music Group und Bertelsmann Music Group (BMG Entertainment).

23 Dass dadurch erhebliche Sicherheitsprobleme entstehen, dass fremde Benutzer faktisch Zugang zu Verzeichnissen in Rechnern haben, die zwar besonders legitimiert worden sind, von denen aus aber der Zugriff zu anderen, nicht legitimierten Verzeichnissen nicht prinzipiell ausgeschlossen werden kann, ist unmittelbar evident. Einmal „inside“, ist es leichter, zu weiteren Informationen zu gelangen, als überhaupt in den Rechner zu kommen. 
mal erworbenen Informationsobjekten an die nächsten Interessierten. Die Frage ist also, was dieses Umschlagen von privatem, in der Wirkung begrenzten Verhalten in öffentliches, global unbeschränktes Verhalten bedeutet.

Der kurzfristige Erfolg von Napster (mit sicherlich einigen hundert Millionen Downloads) beruhte sicher auch darauf, dass die Musikverwertungsindustrie sich nicht darauf eingestellt hatte, ihre CD-ROM-Produkte, z.B. über Verschlüsselungssoftware, so zu sichern, dass keine MP3-Files von Unberechtigten erstellt werden können. Es war zu erwarten, dass das „Paradies“ des freien Austauschs von digitaler Musik nicht dauern kann - zu viel steht für die Informations-, Musik- und Filmindustrie auf dem Spiel. Die Klage von RIAA war nur ein Schritt zur Vertreibung aus dem Paradies. Der „Fall“ (oder der Eintritt in die reale Welt des Kommerz) begann im Oktober 2000 dadurch, dass Napster mit Bertelsmann über ein \$50 Mio. Darlehen eine Allianz eingegangen war.

Bertelsmann hatte als wohl erste Firma der Major record labels ${ }^{24}$ die Bedeutung des neuen Geschäftsmodells erkannt. Thomas Middelhoff, der Bertelsmann-Chef, formulierte dies bei der Ankündigung der Allianz eindeutig: „There's no question that file-sharing will exist in the future as part of the media and entertainment industry .... There's no way to deal with this fact (other) than to develop a business model for file-sharing" (http://news.cnet. com/news/0-1005-200-3345604.html). Trotz der Ankündigung von Napster, bei der Idee des freien Austauschs in der Napster-Community zu bleiben, wird es den entscheidenden Wechsel geben, nämlich den von einer Community zu einem Mitgliedsverein (in der Tradition des alten Bertelsmann'schen Buchklubs). Auf der Grundlage von (niedrig gehaltenen) pauschalen Mitgliedsbeiträgen könnte der Austausch weiter betrieben werden. Mit den Einnahmen könnten Lizenzverträge mit den Unternehmen der Musikproduktion geschlossen werden, damit deren Produkte rechtlich abgesichert getauscht werden können.

Die Musikindustrie hat ihre Lektionen gelernt. Die Allianz mit Bertelsmann war nur der erste Schritt. Mit MusicNet haben sich die großen Herstellerfirmen zusammen mit der Service-Firma RealNetworks (mit fast 200 Mio. Nutzern) eine neue Plattform geschaffen ${ }^{25}$, deren Leistung in erster Linie darin besteht, eine professionelle Organisations- und DRM-Software bereitzustellen, die an Vertriebspartner lizensiert werden kann. ${ }^{26}$ Über MusicNet kann Musik

24 Bertelsmann gehört BMG Entertainment - eine der Firmen, die die Klage gegen Napster angestrengt hatten. BMG hatte nach dem Bertelsmann-Napster-Engagement die Klage natürlich zurückgezogen.

25 http://www.musicnet.com/ - Zu MusicNet gehören: Warner, EMI, BMP und Zomba. Sony und Vivendi setzen bislang auf ihr eigenes Projekt Duet.

26 MusicNet bezeichnet sich als „first digital music subscription platform featuring on-line downloads" (http://www.musicnet.com/Frame_about.html). 
entweder heruntergeladen oder laufend angehört werden (Streamable music). Die Gebühren dafür werden individuell von den Lizenznehmern festgelegt. Napster ist dann, z.B. neben America Online, nur einer von vielen möglichen Lizenzpartnern für MusicNet und kann lediglich darauf vertrauen, dass das aufgebaute Kundenpotenzial auch bei den neuen durch die Allianz mit Bertelsmann entstandenen Konditionen des pauschalen Mitgliedsbeitrags annähernd erhalten bleibt.

Die These der Venterisierung eines Napsterisierungsvorgangs scheint sich zu bestätigen. Der Erfolg der Industrie gegenüber dem „Rebell“ war allerdings in erster Linie durch das letztlich doch zentrale Organisationsmodell von Napster möglich. Zwar wurden Input- und Download-Leistungen verteilt von den Nutzern selber erbracht, aber die Vermittlungsprozesse liefen über Napster als Tausch-Server. Schwieriger zu kontrollieren sind die Tauschvorgänge (und andere Internetanwendungen) nach dem Prinzip des Peer-to-Peer (P2P)Austauschs, wie es z.B. Gnutella, Freenet und viele andere realisieren. ${ }^{27}$

P2P ist im Gefolge der Napster-Auseinandersetzung zum Thema der Öffentlichkeit geworden. ${ }^{28}$ P2P-Netze sind völlig dezentral organisiert und ermöglichen anonyme und verschlüsselte, kostengünstige und leistungsstarke effiziente Speicherung, Übertragung und Nutzung von Informationen ohne die Vermittlung eines zentralen Servers. Die Vermittlung wird durch direkte Punktzu-Punkt-Verbindung zwischen den Teilnehmern geleistet, die in dem jeweiligen P2P-Verbund zusammengeschaltet sind. ${ }^{29}$ Aufgelöst ist also die Idee einer Institution, wie es sie noch bei Napster gab, die die Koordination in der

27 Eine Übersicht über P2P-Anwendungen und Entwicklungen findet man unter http://www.at-web.de/p2p/p2p.htm. Fast schon grotesk und in dem Versuch, neue Umgangsformen mit Wissen zu kriminalisieren, gefährlich hat der Nutella-Hersteller Ferrero mit der Argumentation seiner Klageschrift „Millionen von Internet-Nutzern würden bei dem Wort Nutella nicht mehr an die familienfreundliche Nuss-Nougat-Creme denken, sondern ein virtuelles Konglomerat aus Urheberrechtspiraten und Kinderpornofreunden assoziieren“ Anfang 2001 vor dem Kölner Landgericht Recht bekommen (http://www.chip.de/ news_stories_3706.html). „Gnutella“ darf entsprechend nicht mehr verwendet werden.

28 Mitte Februar gab es eine eigene P2P-Konferenz in San Francisco; vgl. http://www.heise.de/ct/01/06/080/default.shtml=.

29 In der akademischen Umgebung, zunächst als studentische Initiative am M.I.T. ab 12/99, ist vor allem das Free Haven Project bekannt geworden (www.freehaven.net/). Dieses hatte zum Ziel die Entwicklung eines sicheren dezentral organisierten „Datenhafens“, in dem Daten anonymisiert gespeichert werden können und der robust gegenüber „feindlichen“ Zerstörungsangriffen ist. Die Ziele werden gegenwärtig wie folgt formuliert: „Anonymity: We try to meet this goal for all parties: the publishers that insert documents, the readers that retrieve documents, and the servers that store documents. We are in the process of designing and developing a free, low-latency, two-way mixnet for forward-anonymous communication. Accountability: We consider methods for achieving accountability without sacrificing anonymity. In particular, we're researching reputation and micropayment schemes, which allow us to limit the damage done by servers which misbehave. Persistence: The publisher of a document - not the servers holding the document - determines its lifetime. Flexibility: The system functions smoothly as peers dynamically join or leave." 
Hand behält. Gnutella z.B. (oder wie immer es nach der Nutella-Klage heißen mag, s.o.) ist nichts anderes als ein einmal in das Internet freigesetztes Protokoll, dessen sich Nutzer zu welchen Zwecken auch immer bedienen können. Wird die Nutzung mit starker Kryptographie verbunden, wie es bei Freenet zur Anonymisierung der Transaktionen der Fall ist, ist eine externe Kontrolle so gut wie ausgeschlossen.

P2P-Netze, in Verbindung mit frei verwendbaren Verschlüsselungsverfahren, werden vermutlich die Zukunft des Internet bestimmen, gleichermaßen als Möglichkeit - Gefahr und Chance - , ohne Kontrolle den Austausch von Wissen und Information organisieren zu können, aber auch als Möglichkeit Gefahr und Chance -, die Internet-Öffentlichkeit auf in P2P-Netzen organisierte Partikularöffentlichkeiten zu reduzieren. Kommerzielle und politische Kontrolle werden schwieriger.

P2P ist wie jede Technologie zunächst anwendungsneutral - wer sich zu welchen Zwecken in P2P-Netzen zusammenschaltet, ist von der Technologie her nicht bestimmt. Entsprechend wird der P2P-Gedanke, der ursprünglich in „Hacker“-Bewegungen wie Freenet entstanden ist und freie Nutzer,märkte“ wie über Gnutella schaffen wollte, auch kommerzialisiert, z.B. über die Firma Groove Networks mit einer entsprechenden P2P-Groupware (Groove), mit Anwendungen wie synchrone Kommunikation (Chat), gemeinsame Projekte, File Sharing, virtuelle Konferenzen und Arbeitstreffen etc. realisiert werden. ${ }^{30}$ Die Venterisierung (jetzt verstanden als innovative bzw. diffundierende Umsetzung von Verfahren aus der Internet-Basisbewegung) ist nirgendwo aufzuhalten.

\subsection{Napsterisierung von Hollywood - CSS vs. deCSS}

In die Auseinandersetzung um freie Nutzung oder Kontrolle digitaler Objekte werden zunehmend auch digitalisierte Videos einbezogen, auch wenn faktisch Übertragungs- und Speicheraufwand beim Herunterladen von Filmen für „Napsterisierer“ bislang noch restriktiv wirken. Dennoch spricht man seit der Auseinandersetzung um die Dekodierungssoftware für DVD (Digital Versatile Disc)-Produkte schon von der „Napsterisierung von Hollywood“ (http://www.chip.de/news_stories/news_stories_156043.html).

Bei der kommerziellen Nutzung von DVD-Produkten konnte die FilmVideoindustrie von vorneherein einen anderen Weg als bei den CD-ROMs einschlagen, die zunächst weitgehend ungeschützt auf den Markt gekommen sind. DVD wird durch eine Authentifizierungs- und Verschlüsselungstechnik geschützt, die z.B. als CSS (Content Scrambling System) in die offiziellen DVD-Systeme eingebaut ist. D.h. ein DVD-Film ist nur dann lesbar, wenn die

30 Andere P2P-Firmen sind z.B. Battery Ventures. 
originale Scheibe zur Authentifizierung in das Laufwerk bei einem speziell dafür legitimierten Rechner mit dem richtigen Betriebssystem eingelegt ist und entsprechend die verschiedenen Ver-/Entschlüsselungsverfahren aktiviert werden. Andere Verfahren, z.B. für die Online-Nutzung, werden weiter entwickelt. Sie sollen das leisten, was an funktionalen Anforderungen für alle DRM- bzw. IPR-Verfahren ${ }^{31}$ gilt: a) die beteiligten Subjekte (Rechteinhaber, Nutzer) zu identifizieren und zu lokalisieren; b) die zu schützenden Objekte eindeutig zu identifizieren und zu lokalisieren und c) genau zu spezifizieren, in welchem Umfang die Objekte von wem genutzt werden können (Bearman 2000; AAPDRM 2000). Diskutieren wir stellvertretend die CSS-Entwicklung für DVD.

CSS beruht auf Wissen über Verschlüsselung und Authentifizierung, ist in Software kodiertes Wissen. Das Wissen selber ist aber kaum geschützt zu halten. Kein Wunder, dass verschiedene Gruppen sich rasch das Wissen angeeignet haben, wie die entsprechende Software unterlaufen werden kann, und sei es durch auf T-Shirts gedruckte Code-Zeilen. Konsequent kann dann dieses Wissen erneut in Software, also in einem Informationsprodukt, kodiert werden. Darf diese Information, mit der nun gehandelt, also ohne Berechtigung dekodiert werden kann, frei verteilt werden, und darf sie vor allem frei genutzt werden?32 Ist die getroffene Unterscheidung von Wissen und Information(sprodukt) überhaupt sinnvoll, wenn das Wissen, wie CSS entschlüsselt werden kann, öffentlich ist und damit ohne Weiteres zur Information werden kann? Wie kann/darf verhindert werden, dass dieses Wissen als Information, hier also als Informationsprodukt in Form von deCSS, auch verwendet wird?

31 IPR=Intellectual Property Rights, synonym mit DRM=Digital Rights Management

32 Vergleichbare Fragen wurden in der zurückliegenden Kryptographie-Debatte gestellt. Bis Ende der 90er Jahre war die Nutzung starker Kryptographie auch mit Beruf auf das Waffengesetz in den USA verboten, so dass kryptographische Hard- und Software unter die Regelungen von AECA (The Arms Export Control Act) bzw. von ITAR (International Traffic in Arms Regulation) subsumiert und Kryptographie-Exportverbote erlassen werden konnten, vor allem mit Blick auf (damals) starke Kryptographie (oberhalb 40 bits). Gegen den Entwickler von PGP (Pretty Good Privacy), Phil Zimmermann, der PGP, auf der Basis des RSA-Algorithmus und des Public-key-Ansatzes entwickelt, zur freien Verfügung ins Netz gestellt hatte, waren lange Zeit verschiedene Gerichtsverfahren anhängig. Erst 1995 wurde der politische und Verwertungsstreit u.a. dadurch beendet, dass sich eine öffentlich anerkannte Institution, nämlich das M.I.T. bereit erklärte, für PGP eine neue SoftwareLösung vorzulegen. Das geschah dann auch in der Zusammenarbeit von Zimmermann und anderen Experten vom M.I.T. Das M.I.T. sah die öffentliche Verantwortung der Wissenschaft, kryptographische Verfahren einer breiten Öffentlichkeit offen zu halten, und engagierte sich entsprechend. Und schließlich sah sich auch die USA-Regierung 1998/99 durch gemeinsamen Druck der Informationswirtschaft und der InternetBürgerrechtsbewegungen - eine in den USA nicht seltene Koalition - veranlasst, die Beschränkungen auf Kryptographie aufzugeben. Was als kriminell angesehen war - das Unterlaufen von Kryptographie-Bestimmungen durch frei verfügbare Software -, kann zuweilen durch die Praxis in den elektronischen Informationsräumen legalisiert werden. $\mathrm{Ob}$ dies allerdings von dauerhafter Wirkung ist, kann mit Blick auf die seit September 2001 grundlegend veränderte globale politische Lage bezweifelt werden, in der Ziele wie Sicherheit und Kontrolle gegenüber Freizügigkeit und Privatheit dominant werden. 
Rein juristisch, zumindest in den USA, scheint die Lage klar zu sein. Ob nun der Gesetzgeber in den USA die Auseinandersetzung um DVD-Produkte oder andere durch Software geschützte digitale kommerzielle Objekte antizipiert hatte oder nicht, jedenfalls ist die Gesetzeslage in den USA so, dass aufgrund des 1998 vom US-Kongress verabschiedeten „Digital Millennium Copyright Act“" (DMCA) die Erstellung und der Vertrieb von Software unter Strafe gestellt ist, durch die legale Copyright-Ansprüche unterlaufen werden können (DMCA, 17 USC §1201).

Nach einiger Zeit der Nicht-Anwendung dieses Gesetzes schafft seit Mitte 2000 das DMCA in der Auseinandersetzung um die Neuformulierung des Copyright in den elektronischen Medien nun tatsächlich neue Realitäten. Am 17.8.2000 hat der Richter Lewis A. Kaplan vom U.S. District Court in Manhattan dieses Gesetz auf eine Klage von 8 Hollywood-Filmstudios gegen Eric Corley angewendet und entschieden, dass das Herunterladeangebot für die deCSS-Software auf der Website 2600.com von Corley gegen das Gesetz verstößt. ${ }^{33}$ Mit deCSS kann die Authentifizierungs- und Verschlüsselungstechnik (CSS) von DVD-Filmen unterlaufen werden. ${ }^{34}$

Der Richter hat mit seiner Entscheidung auch explizit Stellung in der Debatte genommen, ob Computer Code eine Form der freien Rede sei, die, wie es die Open-Source-Bewegung verlangt, offenzulegen sei, oder ob Code eine Ware

33 Nach Einsprüchen der Open-Source-Bewegung und von Organisationen wie Electronic Frontier Foundation wird letztlich der Supreme Court in den USA über den Vorgang befinden müssen. In der Vergangenheit haben Internet-Bürgerrechtsbewegungen häufig genug vor dem U.S. Supreme Court als letzter Instanz Recht bekommen, allerdings meistens dann, wenn ihre Interessen konform (wenn auch aus unterschiedlichen Gründen) mit den Interessen der Informationswirtschaft waren, so z.B. bei der endgültigen Ablehnung des von der Clinton-Administration eingebrachten und vom Kongress verabschiedeten Communications Decency Act (CDA), durch das Inhalte von Internet-Angeboten bewertet und kontrolliert werden sollten, oder bei der früheren Ablehnung der staatlich verordneten ClipperKryptographie-Software. Bei dem DVD-Problem sind die Interessen der HollywoodIndustrie und die Interessen der Internet-Basisbewegungen konträr.

34 Es ist nicht unwesentlich, sich zu erinnern, wie es zu der Auseinandersetzung um DVD gekommen ist. Verschiedene Gruppen der Open-Source-Betriebssystem-Software LINUX hatten sich darum bemüht, DVD-Produkte auch für LINUX lauffähig zu machen. Eine Gruppe (LSDVD) hatte sich daran gemacht, eine lizensierte DVD-Version zu erstellen, für deren Nutzung ein geringes Entgelt zu bezahlen sei, während eine andere Gruppe (LiViD) an der ursprünglichen Open-Source-Idee festhalten und eine frei zugängliche DVD-Abspielsoftware für LINUX entwickeln wollte. Ziel beider Gruppen war also keineswegs DVD-Piraterie, sondern DVD-Nutzung für LINUX zu ermöglichen. Diese LINUX-Arbeiten hat dann die norwegische Gruppe Master of Reverse Engineering (MoRE) aufgegriffen. Sie hat in der offiziellen Software des DVD-Systems eine Lücke entdeckt, durch die verschlüsselte DVD-Filme aufgeschlossen werden können. MoRE hat dann weiterhin den geheimen CSS-Algorithmus aufgedeckt und veröffentlicht. Dadurch wurde auch deutlich, dass die DVD-Software beträchtliche Mängel hat, was die Open-Source-Bewegung u.a. darauf zurückführt, dass die Software nicht offen in Fachkreisen diskutiert und entsprechend verbessert werden konnte. Die Bereitstellung von DeCSS war also nur ein logischer Schritt. Schlechte Software kann und soll - so ja auch das Ethos der Hacker-Bewegung - durch bessere Software unterlaufen werden. 
wie jede andere sei: In der Begründung heißt es: „Computer Code is not purely expressible any more than the assassination of a politicial figure is purely a political statement". Aus dem generelleren Streit um die Frage, welches Recht höher zu gewichten sei, das Recht auf freie Rede (Publikationsfreiheit) oder das absolute Recht auf den Schutz geistigen Eigentums, hat sich der Richter herausgehalten und hat, wie könnte es auch anders sein, im Prinzip die Berechtigung beider Positionen anerkannt. Bei konfligierenden Grundsatzpositionen gibt es in demokratischen Gesellschaften, so der Richter in seiner Begründung, nur einen Ausweg: „clashes of competing interests like this are resolved by Congress".

Bislang gilt also die explizite Formulierung des DMCA, dass die Erstellung und der Vertrieb von Software untersagt sei, durch die legale CopyrightAnsprüche unterlaufen werden können (DMCA, 17 USC §1201). Wie immer man dies auch interpretieren mag, klar erkennbar ist, dass damit eine Verschärfung der bisherigen Copyright-Regelungen Realität wird, dass also die bislang unter dem Etikett Fair use möglichen Ausnahmen als Grundlage des Umgangs mit geistigem Eigentum eingeschränkt werden. ${ }^{35}$ Es ist offensichtlich, dass zur Zeit die Vertreter der Filmindustrie, besorgt über zunehmende Piraterie in der Form freien Kopierens, das DMCA dahingehend interpretieren, dass der Kongress eine radikale Wende im Verständnis von Fair use vorgenommen habe und dass wegen der Einsatzmöglichkeiten neuer technologischer Kontrollmechanismen Ausnahmen nicht mehr sinnvoll seien. Anders als im früheren Medien können durch diese Verfahren alle individuellen Aktionen mit digitalen Produkten exakt geregelt werden, also z.B. wie viele Personen Einsicht in ein Produkt nehmen können und wie lange sie das dürfen, ob das Produkt weiterverliehen werden darf, ob Exzerpte möglich sind, welches Betriebssystem passend für das Produkt ist, ....

Um diese neuen Ansprüche zu sichern, ist ein ganz neuer Wirtschaftszweig in der Publikationsindustrie entstanden, der, wie erwähnt, unter dem Namen DRM- oder IPR-Software für Content Provider bzw. für Publikationsorganisationen jeder Art anbietet, durch die sie den Vertrieb und die Nutzung ihrer digitalen Produkte unter Kontrolle halten können. Die besprochene CSSSoftware ist Teil dieses Digital Rights Management, hier bezogen auf die Nutzung von DVD-Produkten. DRM greift aber in die Kommerzialisierung und die Kontrolle aller digitaler Informationsprodukte ein. Man kann vermuten, dass ein Bereich der Informationswirtschaft, Ersteller von Software, hier von DRM-Technologie, sich dadurch neue Objektbereiche verschafft hat, dass einem anderen Teil der Informationswirtschaft, den Wissensverwertern,

35 Der Staat, so die allgemeine Meinung, hat nicht das geistige Eigentum absolut geschützt, sondern nur für eine begrenzte Zeit und mit der Intention, Anreize zum Schaffen geistiger Produkte zu setzen, durch die die Gesellschaft als Ganze, zur Förderung von Wissenschaft und Kunst bzw. des intellektuellen Lebens insgesamt, Nutzen ziehen kann. 
Chancen der Kontrolle suggeriert wurden, die real gar nicht vorhanden sind. Durch Software können Besitzansprüche nicht wirklich durchgesetzt werden. Das Setzen auf DRM-Technologie bzw. das Setzen auf Kontrolle (einhergehend mit dem Kriminalisieren von Gegenpositionen) hat das Nachdenken und Entwickeln neuer Modelle beim Umgang mit digitalen Wissensprodukten eher verhindert. Das wird auch an der Diskussion über ebooks erkennbar, wo sich das Beharren auf Kontrollmechanismen als kontraproduktiv zu erweisen scheint.

\subsection{Kontraproduktive Kontrolle von eBooks}

Konflikte um Besitz- und Nutzungsansprüche bzw. um Kontrollmöglichkeiten sind bislang in erster Linie an den besprochenen Beispielen digitalisierter Musik und digitalisierter bewegter Bilder (Videos) manifest geworden. Betroffen sind aber natürlich im Prinzip alle elektronischen Informationsprodukte. Die einfachste mediale Form, Texte in Form von elektronischen Büchern, Ebooks, steht erst am Anfang der Napsterisierung. Der Markt für Ebooks, anders als der für Musik-CD-ROMs oder Video-DVD, entsteht erst. Auch hier ist die Ambivalenz von freier Nutzung und Kontrolle ersichtlich: Der Anspruch auf private persönliche Nutzungsrechte für digitale Produkte, vergleichbar den bisherigen Rechten bei gedruckten Produkten (Mehrfachnutzung, beliebige Weitergabe, Zitieren etc.) wird ebenso erhoben wie auf der anderen Seite der Anspruch auf weitergehende Kontrolle der Nutzung, vor allem aus Sorge vor der ohne weitere Maßnahmen nicht mehr nachvollziehbaren Weitergabe digitaler Produkte in den elektronischen Räumen des Internet.

Auch hier schafft die Anwendung der durch das US-amerikanische Digital Millennium Copyright Act vorgegebenen Rahmenbedingungen neue Realitäten in der Auseinandersetzung um die Kontrolle oder Nutzung digitaler Informationsprodukte. Mitte Juli 2001 wurde der Russe Dmitry Sklyarov verhaftet und für einige Wochen ins Gefängnis gesteckt, weil er auf einer Konferenz (der DefCon Hacker-Konferenz am 16.7.01 in Las Vegas) über offensichtliche Mängel der bei eBooks zum Einsatz kommenden Kryptographie informiert und weiterhin über Software der Moskauer Firma ElcomSoft, bei der Sklyarov arbeitet, berichtet hatte, mit der in Teile der Adobe-Verschlüsselungssoftware für eBooks eingegriffen werden kann. Das FBI hatte auf die Anzeige von Adobe reagiert, weil das Publikmachen der Arbeiten der Moskauer Firma auf der Konferenz in den USA gegen das DMCA verstößt. Amerikanische Electronic Rights Groups wie Electronic Frontier Foundation protestierten entsprechend heftig gegen diese weitere Anwendung des DMCA und sehen darin, wie in der Einschränkung der CSS-Software, eine Bedrohung 
des bisherigen Fair use bei der Benutzung von gedruckter Information. ${ }^{36}$ Durch die ElcomSoft-Software, die wie die deCSS-Software außerhalb der USA erworben und benutzt werden kann, werden Restriktionen beseitigt, die exklusive Rechte an der Nutzung von eBooks nur dem einräumen, der das eBook erworben hat. Mit der ElcomSoft-Software - und dies ist nur ein Beispiel für viele vergleichbare Napsterisierungs-Software - ist es z.B. möglich, für den eigenen Gebrauch von Adobe-eBooks eine Backup-Kopie zu machen, aber auch das eBook auf einem anderen Computer im eigenen Haus zu übertragen oder eine Kopie für einen Freund zu erstellen - alles einfache Dinge, die bislang beim Kauf eines gedruckten Buches unkritisch waren, durch fortgeschrittenes DRM bzw. IPR aber kontrolliert und verhindert werden können. Auch nützliche Anwendungen, dass Blinde sich mit ihrer Software eBooks elektronisch vorlesen lassen können, werden durch eBook-Restriktionen verhindert, es sei denn, sie sind in den DRM-Spezifikationen explizit formuliert. Solche Anwendungen sind nur möglich, wenn die eBooks in eine andere Software-Umgebung geladen werden, als sie von den Herstellern vorgesehen ist. Das ist aber das Mindeste, was von eBooks verlangt werden kann, nämlich dass man sie in seine eigene Umgebung zur freien weiteren persönlichen Nutzung herunterladen kann. Das ist nicht nur der eine Rechner mit dem einen Betriebssystem, sondern in der Zukunft eine Vielzahl elektronischer, auch mobil zu nutzender Geräte, auf denen man seine ebooks lesen will. Venterisierer sehen aber darin genau den Weg offengelegt zu einer Napsterisierung der eBooks. Wenn die Nutzungsumgebung nicht mehr kontrolliert werden kann, könnten auch eBooks freizügig in entsprechende Verteil-Server oder P2P-Dienste eingebunden werden. Nicht zuletzt deshalb werden zur Zeit auch über DRM hinausgehende Kontrollmöglichkeiten, wie die Anwendung von Kopierschutztechniken auf PCn, erwogen.

Offensichtlich verschärfen sich die Spannungen zwischen denjenigen „who need and use information and those who want to control it" (Los Angeles Times 6.8.2001, A11). Fraglich allerdings, ob in der Kombination von verschärfter rechtlicher Regelung und Softwaremaßnahmen des DRM der Erfolg von Produkten wie eBooks gesichert werden kann, die ohnehin bislang nur einen minimalen Verkaufserfolg erzielen konnten. Der Grund für den bisherigen Misserfolg wird zum einen darin gesehen, dass eBooks nicht auf reale Bedürfnisse von Lesern abgestimmt zu sein scheinen, die bislang mit den gedruckten Produkten zufrieden sind. Die Zurückhaltung wird aber auch darauf zurückgeführt, dass der technische Umgang mit den eBooks noch als zu kompliziert und unzuverlässig angesehen wird und vor allem die Nutzungsbedingungen, die über die Software der eBooks geregelt werden, als zu restrik-

36 Viele weitere Links, zusammengestellt von der Electronic Frontier Foundation, zu dem aktuellen Fall Sklyarov und die Folgen unter http://www.eff.org/IP/DMCA/US_v_Sklyarov/. 
tiv empfunden werden. Das Vertrauen in die Zuverlässigkeit und Angemessenheit von DRM-Techniken ist durchaus nicht auf den elektronischen Märkten gegeben (vgl. Cheng/Litva/Main 2001), und Vertrauen ist die Grundlage kommerziellen Erfolgs (Kuhlen 1999).

In dieser Situation scheint eine Verschärfung der Nutzungsbedingungen eine klassische Verlierer-Situation zu generieren. Kontrollmechanismen sind vermutlich eher kontraproduktiv: Verlage versuchen, ihre Umsätze zu kontrollieren und zu steigern, indem sie die Nutzung restringieren und eng an die Vertragsbedingungen binden, und genau diese mangelnde Freizügigkeit verhindert (neben anderen Faktoren) die Entwicklung eines offenen und größeren Marktes. Neue Modelle der Nutzung sind eher gefragt als Modelle der erzwungenen Kontrolle. ${ }^{37}$

\subsection{Napsterisierung in Ausbildung und Wissenschaft}

Bevor wir aus der vorhergehenden Diskussion einige Schlussfolgerungen ziehen, wollen wir noch auf eine weitere Ambivalenz im Napsterisierungsbegriff eingehen, und zwar aus dem Umfeld der Wissenschaft, wo Napsterisierung durchaus negativ als Plagiatisierung verstanden werden kann, aber auch als neue Formen der Direktpublikation unter Umgehung der Venterisierungsverfahren der bisherigen Distributoren der wissenschaftlichen Produktion.

\section{Referenzverluste}

Mit „Napsterisierung“ wird weitergehend als das Umgehen von Verwertungsrechten die Einstellung bezeichnet, für Wissensprodukte nicht nur nicht zu bezahlen, sondern sich diese anzueignen, ohne auf die Urheber zu referenzieren. So zu tun, als sei die Wissensproduktion anderer die eigene, wird im allgemeinen als Plagiat bezeichnet ${ }^{38}$, in Ausbildungsumgebungen als Betrug. Robert S. Boynton weist in einem Artikel in der Washington Post ${ }^{39}$ auf diese Napsterization of knowledge in der akademischen Ausbildung hin, die seit einigen Jahren breit um sich greife. Gemeint ist dabei ,the notion that ideas (like music) are little more than disembodied entities, out there in the ether, available to be appropriated electronically in any way users wish. As a result, the line between the hard-won insights that are produced by solitary scholarship, and the quotidian conclusions that collaboration yields, has been blurred. What now constitutes honorable behavior is an open question." Napsterisierung von Wissen bedeutet in diesem Verständnis also das durch

37 Vgl. Manasse (2000): „Digital rights management based on enforcement is moribund.“

38 Wir werden sehen, dass im allgemeinen Zusammenhang der Hypertextifizierung von Wissen der generelle Ausdruck „Plagiat“ als Übernahme von Wissensobjekten oder Teilen von ihnen, ohne ihre Fremdreferenz anzugeben, problematisch wird.

39 Washington Post, Sunday, May 27, 2001; Page B01 - http://www.washingtonpost.com/ ac2/wp-dyn/A80312-2001May26?language= printer 
die elektronischen Medien leicht gemachte Unterlaufen des bisherigen Wertesystems der Ausbildung, das Betrug (Cheating) allgemein und Plagiat speziell außerhalb des akademischen Ethos stellt.

Was folgt daraus, und wie soll sich das akademische System dagegen wehren? Boynton sieht in dieser "napsterization of knowledge“ auf dramatische Weise den in amerikanischen Universitäten vielerorts formulierten Honor code gefährdet, der Betrug bzw. das Verhalten, sich auf unredliche Weise Vorteile gegenüber anderen Studierenden zu verschaffen, als unethisch brandmarkt und entsprechend scharfe Sanktionen bei entsprechenden Verstößen nach sich zieht. Für die gesamte Diskussion um die Abwehr von Napsterisierung ist einschlägig, dass sich Boynton dagegen wendet, der Napsterisierung von Wissen dadurch auf die Spur zu kommen, dass entsprechende Software eingesetzt wird. Solche Software wurde inzwischen verschiedentlich entwickelt, um das Abschreiben bei anderen Studierenden oder das unreferenzierte Verwenden von publizierten Materialien aufzudecken. ${ }^{40}$

Software zur Sicherung des Honor-Code-Prinzips geschieht nach der Devise „Trust, but verify“, und dagegen wendet sich Boynton: „Neither the Napsterization of knowledge nor the honor code scandal will be solved through technological means alone. To go down that road guarantees a never-ending 'arms race'“. Gegen die technologische Lösung, die, da immer wieder durchbrochen, immer stärkere Mittel einsetzen muss, setzt Boynton das regulative, faktisch wohl immer wieder nicht eingehaltene ethische Prinzip „Trust, but don't verify“, wobei in Kauf genommen wird, dass der Missbrauch immer vorkommen wird, aber ethisch nicht akzeptabel ist und von der wissenschaftlichen Gemeinschaft nicht akzeptiert wird. Der bloße Appell wird wohl nicht reichen. Vielmehr müssen Vorbilder - die akademischen Lehrer selber müssen Wissensreferenzierungen und Schutz von reklamiertem Eigentum einhalten und natürlich Anreize geschaffen werden, dass das Einhalten von Vertrauen sich mehr „lohnt“ als dessen Missbrauch.

Eine konstruktive Lösung, mit der „Napsterisierung“ auch in diesem Fall positiv umgedeutet wird, wird aus der Open-Source-Bewegung angeboten. Die Lizensierungsregeln für Open-Source-Software wird gegenwärtig durch entsprechende Regelungen auf die textuellen Dokumentationen der Software und dann allgemein auf Texte jeder Art übertragen. In der Praxis bedeutet das, dass Autoren von Netzpublikationen ihren Text mit einem Lizensierungsvermerk versehen (können) ${ }^{41}$, auf Grund dessen entsprechend der Tradition des Copyleft jedermann diesen Text frei verwenden und modifizieren kann, unter der Bedingung, dass weitere Nutzungen (bis hin zu Zitierungen) und Modifikationen ebenfalls frei zugänglich gemacht werden und dass jede Nutzung

40 Z.B. über http://www.plagiarism.phys.virginia.edu

41 GNU Free documentation license - http://www.fsf.org/copyleft/fdl.html 
und Modifikation (einschließlich von Übersetzungen) referenziert werden muss.

\section{Selbstorganisation als Reaktion auf Marktversagen}

Das mit Napster verbundene Organisationsmodell wird mit dieser Bezeichnung verschiedentlich auch für eine Form der Selbstorganisation von Wissenschaft bei der Publikation von und dem Zugriff auf digitale(n) Wissensobjekten verwendet (vgl. Sietmann 2001). ${ }^{42}$ Es ist nicht zuletzt die fortschreitende Venterisierung auch der wissenschaftlichen Produktion, die Wissenschaftler veranlasst hat, sich um die Publikation und Verteilung ihrer Produktionen selber zu kümmern. Dies auch deshalb, weil klassische Verlagsleistungen, wie Qualitätskontrolle und Öffentlichkeitsarbeit, zunehmend von der Wissenschaft selber wahrgenommen werden (müssen). Nur noch wenige Verlage leisten aktive Akquirierungsarbeit. Die meisten Verlage verlangen von den Autoren druckfertige Manuskripte, und die Qualitätskontrolle über Peer-Reviewing geschieht ohnehin durch Wissenschaftler selber. Zudem ergeben sich bei Verlagspublikationen immer größere Zeitverschiebungen zwischen der Fertigstellung der Forschung bzw. der Erstellung der Manuskripte und der Veröffentlichung in traditionellen aber auch elektronischen Journalen. Weiter ist eine Präsenz-Vollständigkeit von Verlags-Zeitschriften in den Bibliotheken selbst bei engeren Wissensgebieten bei fallenden Bibliotheksetats und restriktiver Preispolitik der Verlage auch für E-Produkte kaum noch möglich. Ob das nun eine Bibliotheks- oder eine Verlagskrise ist, sei dahingestellt - auf jeden Fall wird in der gegenwärtigen Praxis der Widerspruch zwischen Leistung und Venterisierungsanspruch deutlich, und es stellt sich zunehmend die Frage, mit welchem Recht Wissensverwerter von Wissenschaftlern bzw. von den Institutionen, die den Informationsbedarf von Wissenschaftler absichern sollen (Bibliotheken etc.), den Rückkauf ihrer eigenen Produkte verlangen können, ohne dass Mehrwertleistungen offensichtlich sind. Kommen hinzu die den Wissenschaftlern zur Verfügung stehenden technischen Möglichkeiten durch die neuen elektronischen Medien für Produktion, Bereitstellung, Verteilung und Nutzung von Informationsprodukten, so entwickeln sich fast zwangsläufig neue Organisationsmodelle für den Umgang mit Wissen und Information auch in der Wissenschaft.

Entsprechend stellen weltweit immer mehr Wissenschaftler ihre Texte mit aktuellen Forschungsergebnissen ins Netz, sei es direkt persönlich über die eigene Website oder sei es über Pre-Print-/e-Server von Verbänden oder anderen

42 Im wissenschaftlich spielerischen Bereich wird das verteilte Modell der Ressourcennutzung z.B. von SETI@home für die Suche nach extraterrestrischer Intelligenz verwendet. Bislang haben über 3,23 Millionen Personen (Stand 08/01) ihre Rechnerkapazität zur Verfügung gestellt, um die Ziele des Projektes zu unterstützen. Interessanter ist hier eher die Organisation der verteilten Ressourcen als der tatsächlich damit erzielte Erfolg. 
Fachorganisationen ${ }^{43}$ oder Publikations-Server, wie sie inzwischen von vielen Bibliotheken oder Rechenzentren aufgebaut werden.

Allerdings werden entsprechend den jeweiligen lokalen oder organisationsspezifischen Gepflogenheiten unterschiedliche Formate und vor allem heterogene Metadaten zur Beschreibung der Inhalte der Texte oder anderer digitaler Objekte verwendet. In den wissenschaftlichen Bibliotheken selber ist weder im nationalen Bereich (in Deutschland) geschweige denn im internationalen Bereich auch nur annähernd eine Angleichung oder Standardisierung von Metadatensprachen bzw. -formaten erreicht worden. Entsprechend unbefriedigend, sowohl was Vollständigkeit (Recall) als auch was Genauigkeit (Precision) angeht, fallen die Ergebnisse der weltweit operierenden Suchmaschinen aus. Deren Retrieval kann nur so gut sein wie die Indexierung (Inhaltsbeschreibung) der Ausgangsmaterialien. Die Indexierungsqualität der Suchmaschinen kann nicht befriedigend ausfallen, wenn sie sich lediglich auf die in den Objekten enthaltenen Informationen bzw. auf die von den Anbietern verdeckt, also nur für die Suchmaschinen einsehbaren Informationen abstützen. Erforderlich sind kontrollierte Vokabularien bzw. allgemein anerkannte formale Metadatenstrukturen zur Beschreibung der Inhalte. ${ }^{44}$ Es ist also nicht zu verkennen, dass die Formen der Direktpublikationen zu einer dramatischen neuen Unübersichtlichkeit geführt haben. Entsprechend werden auch seit einigen Jahren entsprechende Maßnahmen entwickelt, z.B. über die Open Archives Initiative ein, die in der verteilten Organisation als ein neues positives Napsterisierungsmodell verstanden werden kann.

Die Open Archives Initiative (OAI) versucht seit 1999 durch eine Erweiterung des HTTP (Transaktionsprotokoll für das World Wide Web) eine neue Übersichtlichkeit in das skizzierte „Chaos“ der wissenschaftlichen Direktpublikation zu bringen. Dieses OAI-Protokoll (Open Archives Initiative Protocol for Metadata Harvesting) verlangt die Verwendung von sogenannten Persistent identifiers zum eindeutigen Nachweis sowohl des Archivs als auch der Dokumente innerhalb des jeweiligen Archivs. Entscheidender aber ist, dass den Wissenschaftlern ein formaler Metadatensatz angeboten wird, mit denen sie die Inhalte ihrer Objekte entsprechend festgelegter Minimalanforderungen beschreiben und so über Suchmaschinen auffindbar machen können. Das Protokoll stützt sich auf das Dokumentformat XML sowie auf den 'Dublin Core' $\mathrm{ab}$, der sich zu einem Quasi-Standard für Metadaten entwickelt. Es können aber innerhalb der OAI-Struktur andere Wissensklassifikationsformen, wie sie

43 Z.B: das e-Print-Archiv für die Fachgebiete Physik, Mathematik und Informatik, unterstützt von der U.S. National Science Foundation, dem U.S. Department of Energy und betrieben vom Los Alamos National Laboratory - http://arXiv.org/.

44 Metadatenstrukturen sind nicht zu verwechseln mit den Meta-Tags von HTML, die ebenfalls der (allerdings weitgehend unkontrollierten) Inhaltsbeschreibung dienen. Metadatenstrukturen sind semantisch kontrollierte Fach- oder Universal-Ontologien. 
in den einzelnen Disziplinen entwickelt sind, verwendet werden. ${ }^{45}$

Der organisatorische Lösungsvorschlag auf der Grundlage des OAI-Protokolls ist dem der Internet-Tauschbörse Napster vergleichbar, indem kein zentraler Wissenschaftsserver angestrebt wird, sondern eine verteilte Lösung, bei der also das Auffinden und der Zugriff auf einzelne Objekte in einem System der weltweit verteilten Datenhaltung organisiert wird. Weiterhin beruht die Leistung des Systems auf der Bereitschaft der einzelnen Telnehmer (dort der Musikfreunde, hier der Wissenschaftler) ihr Wissen über entsprechendes Metawissen bzw. zunächst einmal über schiere Digitalisierungen der entsprechenden Objekte mit anderen zu teilen. Information Sharing beruht auf dem Do ut des, nicht alleine darauf, dass andere, z.B. Bibliotheken, Informations- und Dokumentationseinrichtungen oder zentrale e-Print-Server die Erschließungsund Vermittlungsarbeit übernehmen. ${ }^{46}$ Vermutlich werden kommerzielle Wissensverwerter im Bereich Wissenschaft sehr bald auch die ökonomischen Vorteile des Prinzips des Information Sharing und der verteilten Organisation erkennen, so wie es Bertelsmann mit dem Napster-Engagement getan hat. Gegenwärtig verharren jedoch die meisten Venterisierer der Wissensverwertungswelt eher bei den klassischen Organisations- und Abrechnungsmodellen, so dass Napsterisierungsverfahren aus der Wissenschaft selber fast unvermeidlich sind.

45 Technisch sind die Fragen der Anwendung des OAI-Protokolls bezüglich Beschreibung und Suche bzw. Anwendung in den Web-Browsern gelöst. Der Erfolg der verteilten „NapsterSciene"-Lösung hängt natürlich davon ab, inwieweit Wissenschaftler bereit sind, die gewisse Mehrarbeit zur Beschreibung ihrer digitalen Objekte in Kauf zu nehmen. Hier kommt den wissenschaftlichen Verbänden eine Aufklärungsaufgabe zu. In Deutschland unterstützt z.B. die Initiative für NetzwerkInformation (DINI) die Verwendung des OAI-Protokolls (vgl. den entsprechenden Appell des DINI für den Einsatz des OAI; http://www.dini.de/dokumente/ oai-appell-0912.pdf). Im DINI sind Organisationen wie AMH (Arbeitsgemeinschaft der Medienzentren der deutschen Hochschulen), IuK (Information und Kommunikation der wissenschaftlichen Fachgesellschaften in Deutschland), ZKI (Zentren f. Kommunikation und Informationsverarbeitung in Lehre und Forschung e.V.) sowie der DBV (Deutscher Bibliotheksverband Sektion 4: Wissenschaftliche Universalbibliotheken) organisiert, so dass, vor allem über den IuK ein Großteil der Wissenschaften direkt erreicht wird.

46 Sicherlich werden diese „zentralen“ Vermittlungsmodelle auch weiter bestehen. In der Wissenschaft nahm das von dem Elementarteilchenphysiker Paul Ginsparg vor zehn Jahren am Los Alamos National Laboratory in New Mexico eingerichtete elektronische Zentralarchiv für das Gebiet der Astrophysik und anderer Teilgebieten der Physik eine Vorbildrolle ein. Los Alamos alleine hat derzeit (Mitte 2001) wöchentlich fast 1 Mio Zugriffe; das es dazu ein Dutzend gespiegelter Server weltweit gibt, schätzt die wöchentliche Zugriffsrate auf ca. 5 Mio. Trotz dieses Nutzungserfolges der zentralen (Physik-)Lösung wird von Ginsparg selber eingeräumt, dass dadurch höchstens $20 \%$ der relevanten Physik-Veröffentlichungen erfasst werden (vgl. Sietmann 2001). Ob eine verteilte Lösung unter Anwendung von QuasiStandards wie dem OAI-Protokoll zu besseren Abdeckungsraten führt, wird, wie gesagt (s. Anm. 45), von der Akzeptanz der einzelnen Wissenschaftler abhängen. Der Nutzungsvorteil ist hier, anders als bei der originalen Napster-Börse, ja nicht unmittelbar erkennbar. 


\section{Schluss}

Unter den Begriffen der Napsterisierung und Venterisierung werden grundlegende Konflikte der gegenwärtigen Informationsgesellschaft ausgetragen, die theoretisch nur durch eine neue umfassende politische Ökonomie von Wissen und Information und praktisch nur durch eine umfassenden Interessenausgleich und einen öffentlichen Diskurs der beteiligten Gruppen gelöst werden können. Es sind die folgenden Interessengruppen auszumachen:

- die Urheber/Autoren von geistigen Produkten selber

- Institutionen, die im Auftrag von Autoren (oder pauschal für diese) die Rechte von Urhebern gegenüber Verwertern von urheberrechtlich geschützten Objekten wahrnehmen (Beispiele: Verwertungsgesellschaften, Schriftstellerverbände, Gewerkschaften wie ver.di)

- Institutionen, in denen Urheber zum Zwecke der Produktion von geistigen Produkten beschäftigt sind (Wissenschaftliche Einrichtungen, private Forschungseinrichtungen, Medienunternehmen mit eigenen Wissensproduzenten)

- Primärverwerter von urheberrechtlich geschützten Objekten, die sich in der Regel über Vertrag die Rechte an der Verwertung gesichert haben (Beispiele: Verlage für gedruckte und elektronische Produkte, Medienunternehmen im Rundfunk- und Fernsehbereich) und Organisationen zur Sicherung deren Interessen (z.B. Börsenverein des Deutschen Buchhandels)

- Sekundärverwerter von urheberrechtlich geschützten Objekten bzw. von Objekten, für die Verwertungsrechte durch Dritte bestehen (Beispiele: Herausgeber von Sammelwerken, Internetdienste, Content Provider, OnlineNewsletter) - dies sind zum Teil natürlich auch Primärverwerter

- Vermittlungseinrichtungen, einschließlich ihrer Verbandsorganisationen, deren Aufgabe es ist, Informationsprodukte für ihre Klientel zu akzeptierbaren Bedingungen bereitzustellen ${ }^{47}$

- Staatliche, zwischen-/überstaatliche und Nicht-Regierungs-Einrichtungen mit Gesetzgebungs- und Ordnungs-/Regulierungsfunktionen, die einerseits die Aufgabe haben, den Vertretern eines gewichtigen Teils der Gesamtwirtschaft (hier der Wissensverwertungsindustrie) sichere Rahmenbedingungen für ihre Geschäfte zu schaffen, andererseits aber auch dem öffentlichen Interesse an einer weitgehend freien, zumindest aber zu fairen Bedingungen möglichen Nutzung von Wissen und Information zu entsprechen (Beispiele: Justizministerien der Staaten, EU-Direktionen, WIPO, UNESCO, ITU)

- Bürgerrechtsbewegungen, Initiativen der „Hacker-Welt“, die sich zunehmend als Gegenpositionen zur Venterisierung/Kommerzialisierung von Wissen und

47 Beispiele: Fachinformationseinrichtungen, Bibliotheken jeder Ausrichtung, Archive, Deutsche Gesellschaft für Informationswissenschaft und Informationspraxis e.V. (DGI http://www.dgd.de/frame.html); Bundesvereinigung Deutscher Bibliotheksverbände e.V. (BDB - http://www.bdbverband.de/index2.html) 
Information verstehen und sich für einen freien Umgang mit Wissen und Information einsetzen

- die Öffentlichkeit bzw. die Endnutzer von geistigen Produkten (im Prinzip also jedermann)

Es ist abzusehen, dass die Auseinandersetzung um Napsterisierung und Venterisierung weitergehen wird. $\mathrm{Ob}$ das ein nicht endendes Hase-und-Igel-Spiel werden wird - bestehende Schlupflöcher für einen nicht kontrollierten Umgang mit Wissen und Information werden mit neuen Verfahren (z.B. intensiviertes DRM) oder ganz neuer Technologie gestopft, die kaum, dass sie auf dem Markt sind, mit neuen Verfahren und neuer Technologie unterlaufen werden, so dass auch diese Löcher gestopft werden müssen, und zwar wahrscheinlich unter Einsatz der gleichen Verfahren und der gleichen Technologie, die die früheren gestopften Löcher durchlässig gemacht haben, etc. etc. oder ob doch dauerhafte Lösungen für einen Informationsfrieden gefunden werden, ist heute noch nicht abzusehen.

Welche Optionen sind vorhanden? Wie kommt man aus dem (hier konstruierten) Gegensatz von Napsterisierung und Venterisierung heraus? Es wird keinen Informationsfrieden geben, wenn bestehende Urheberrechts/Copyrights- bzw. Urheberrechtsverwertungsregelungen, für die es in der „alten" Informationswirtschaft einen breiten Konsens gegeben hat, auf den Umgang mit Wissen und Information in elektronischen Räumen einfach übertragen werden. Das wird weder den Interessen der Venterisierer, die ihre Produkte effizienter vermarkten wollen, noch den Interessen der Napsterisierer, die diese Regelungen unter den neuen medialen Bedingungen für obsolet halten, gerecht.

Erst recht wird es keinen Informationsfrieden geben können, wenn Urheberrechts-/Copyrights- bzw. Urheberrechtsverwertungsregelungen einseitig zu Gunsten der Interessen der neuen Informationswirtschaft verschärft werden. Es sind offensichtlich ganz neue Wege zur Lösung der Copyright/Urheberrechtsfrage im digitalen Medium nötig, die dem Rechnung tragen können, dass einerseits mit Informationsprodukten Geld verdient werden kann, andererseits die freie Nutzung von Information unter fairen Bedingungen weiterhin gestatten. Weder die Verschärfung der bisherigen Schutzrechte noch eine Verschärfung der DRM-Technologie sind dauerhaft eine Lösung. Und erst recht ist es keine Lösung, wenn bestimmte nationale Gesetzgebungen, wie das DMCA in den USA, den globalen Informationsmärkten übergestülpt werden. Wie den elektronischen Räumen angemessene Lösungen allerdings aussehen werden, ist weiterhin offen.

Allerdings sieht es gegenwärtig so aus, dass, zumindest in den USA, die von Lawrence Lessig prognostizierte verschärfte Kontrolle über Wissen dadurch Realität wird, dass Softwareregelungen der Wirtschaft und rechtliche Vorgaben 
der Politik parallel gehen, dass also Politik und Wirtschaft an dem gleichen Strang ziehen. Dies wird dadurch deutlich, dass nicht etwa eine Abschwächung des DCMA zu erwarten ist, sondern eher eine Verschärfung: Entsprechend einer Initiative des US-Senators Fritz Holling „wird [zur Zeit - Mitte 2001 - RK] ein Gesetz vorbereitet, das den Einbau von Kopierschutztechnik in sämtliche PCs und Geräte der Consumer-Elektronik erzwingen soll“" (http://www.heise.de/newsticker/data/cp-09.09.01-003/). Nach diesem Security Systems Standards and Certification Act (SSSCA), wenn es denn Realität wird, darf es keine Computerinstallation mehr geben, die keine zertifizierte Kopierschutztechnik enthält. Ebenso soll in Fortführung des DCMA unter Strafe gestellt werden, wenn Dateien, aus denen der Kopierschutz entfernt wurde, vertrieben oder wenn Computer verkauft werden, bei denen der Kopierschutz unwirksam ist. Es sieht so aus, dass sich die Erwartung der Informationswirtschaft, zunächst in den USA, aber sicherlich mit Konsequenzen für die globale Informationswirtschaft, zumindest in einer mittleren Perspektive erfüllen wird, den gesamten Prozess der Herstellung, Verteilung und Nutzung von Informationsprodukten kontrollieren zu können. Bezog sich diese Gesetzesinitiative wohl zunächst auf den Schutz von Software-Produkten, so dass sie den Interessen der Computerindustrie entgegenkommt, so wird sie, in Radikalisierung des bisherigen Digital Rights Management, auf elektronische Produkte jeder Art Anwendung finden. Das ist keine Grundlage für einen Informationsfrieden. ${ }^{48}$ Eine künstliche Verknappung von Wissens- und Informationsgütern, die die Potenziale gegenwärtiger Technologien einschränkt, war noch nie eine wirtschaftliche Lösung und wird entsprechend auch nicht dauerhaft tragfähig sein.

Nimmt man entsprechend die Interessen der Venterisierer ernst - und das muss man, wenn man anerkennt, dass über Wissens- und Informationsprodukte der größte Teil des Bruttosozialproduktes erzielt wird -, so wird es auch keinen Informationsfrieden geben, wenn keine neuen Geschäfts- und Organisationsmodelle entwickelt werden, auf deren Grundlage investitionsin-

48 Dieser Artikel wurde kurz nach den Terrorakten von New York und Washington fertiggestellt. In der entstehenden öffentlichen Diskussion um das SSSCA, z.B. im Heise-Forum (s.o.), wird der Zusammenhang zwischen dem Globalanspruch der westlichen (USA-) Wirtschaft und dem Globalanspruch der westlichen Informationswirtschaft mit den entsprechenden Konsequenzen des radikalisierten Widerstandes durch Menschen und Kulturen mit anderen Wertvorstellungen durchaus angesprochen. Die Herausforderung an die Politik besteht entsprechend heute weniger darin, die Interessen der Informationswirtschaft durch entsprechende Gesetzgebung zu unterstützen, sondern zu verhindern, dass die Gruppen und Personen aus der angesprochenen Hacker-Welt (dies, wie gesagt, als Metapher für alle Organisationen und Personen, die den freizügigen, d.h. nicht durchgängig kommerziell bestimmten Umgang mit Wissen und Information, für das höchste zu verteidigende Gut halten) in solche Außenseiter- oder sogar kriminelle Positionen gedrängt werden, aus denen dann Handlungen unvermeidbar werden, die einen Informationsfrieden dauerhaft unmöglich machen. 
tensive Mehrwertleistungen zur Erstellung und zum Vertrieb von Informationsprodukten nicht zu einem befriedigendem Return of Investment und entsprechenden Gewinn ermöglichenden Einnahmen führen können. Diese neuen Geschäfts- und Organisationsmodelle werden sich nicht auf eine künstliche Verknappung der Güter Wissen und Information stützen können - ganz im Gegenteil, es ist zu erwarten, dass Märkte umso größer und wirtschaftliches Handeln mit Wissen und Information umso erfolgreicher sein werden, je offener, freizügiger und flexibler die Nutzung von Wissen und Information betrieben werden kann. ${ }^{49}$ In diesem Kontext bekommt die Rede vom Free flow of information und vom Free access, festgeschrieben in so gut wie allen universalen Deklarationen, Konventionen supranationaler Organisationen (UNO; UNESCO, EU - jüngst die EU-Charta, Europarat etc.) und Verfassungen demokratischer Staaten, eine neue Bedeutung. „Frei“ muss nicht „kostenlos“ heißen. Im Französischen wird zwischen „libre“ und „gratuit“ unterschieden. In elektronischen Räumen wird freier Umgang mit Wissen und Information „frei von erzwungener Kontrolle“ bedeuten. Wissen, in digitalen Informationsprodukten repräsentiert, kann nicht eingesperrt werden. Besser man akzeptiert dies und entwickelt auf den Prinzipien des Information Sharing, der verteilten Informationsarbeit, der Lizensierung von Information mit der Verpflichtung der Offenlegung und Referenzierung, des Pricing for Information und der Bereitstellung informationeller Mehrwerte neue Organisations- und Nutzungsmodelle, als dass Napsterisierung und Venterisierung Positionen eines fortgesetzten Informationskrieges besetzen.

\section{Literatur}

AAP-DRM (2000) - Digital Rights Management for eBooks: Publisher Requirements. American Association of Publishers, November 2000 (http://www.publishers.org/home/drm.pdf)

Bearman, David (2000): Functional requirements for IP management. W3C Workshop on DRM, January 2001 (http://www.w3.org/2000/12/drm-ws/pp/archimuse-bearman.html)

Castells, Manuel (2001): Epilog zu Himanen (2001)

Cheng, Spencer; Litva, Paul ; Main, Alec (2001): Trusting DRM Software. W3C Workshop on DRM, January 2001 (http://www.w3.org/2000/12/drm-ws/pp/cloakware.html)

Himanen, Pekka (2001): The hacker ethic and the spirit of the information age, New York (mit einem Prolog von Linus Torvalds und einem Epilog von Manuel Castells)

Kuhlen, Rainer (1995): Informationsmarkt. Chancen und Risiken der Kommerzialisierung von Wissen, Konstanz

Kuhlen, Rainer (1999): Die Konsequenzen von Informationsassistenten. Was bedeutet informationelle Autonomie oder wie kann Vertrauen in elektronische Dienste in offenen Informationsmärkten gesichert werden? Frankfurt a. M.

Kuhlen, Rainer (2000): Wissen als Eigentum? Wie kann der freie Zugang zu den Ressourcen des Wissens in globalen Informationsräumen gesichert werden. Vortrag Tagung der Heinrich-Böll-

49 „IDC [ein führendes Marktforschungsunternehmen] believes the winners will find ways to work with prospective customers, while losers will attempt to force their ecustomers to follow well-worn distribution strategies of business's brick-and-mortar past" (http://www.idc. com/ebusinesstrends/ebt20010830.htm). 
Stiftung 20.21.10.2000 „Wem gehört das Wissen? Geistiges Eigentum in Zeiten des Internet“ (http://www.ib.hu-berlin.de/ kuhlen/Vortraege00-Web/boell201000.pdf)

Kuhlen, Rainer (2001): Universal Access - Wem gehört das Wissen? Gut zu wissen - links zur Wissensgesellschaft. Kongress der Heinrich-Böll-Stiftung. 4.-6. Mai Humboldt-Universität zu Berlin (http://www.ib.hu-berlin.de/ kuhlen/Vortraege01-Web/publikationstext.pdf)

Kurzweil, Ray (1999): The age of spiritual machines: When computers exceed human intelligence. New York, NY

Lessig, Lawrence (1999): Code and other laws of cyberspace, New York, NY

Moravec, Hans (1988): Mind children. The future of robot and human intelligence, Cambridge, MA, London

Manasse, Mark S. (2000): Why rights management is wrong (and what to do instead). W3C DRM Workshop 2001 (http://www.w3.org/2000/12/drm-ws/pp/compaq.html)

Mooney, Stephen (2000): Digital object identifiers for ebooks: What are we identifying? W3C Position Paper (http://www.w3.org/2000/12/drm-ws/pp/pye-brook-mooney.html)

Sietmann

, Richard (2001): Napster für die Wissenschaft Ein neues Protokoll für die virtuelle Fachbibliothek, in: c't 6/2001, S. 78: (http://www.heise.de/ct/01/06/078/)

Stock, Wolfgang (2000): Informationswirtschaft. Management externen Wissens, München, Wien

Werner, Stephan: Napster - quo vadis? 2001 In: NETHICS-Forum (http://www.nethics.net/ nethics/de/brisant/brisant.html)

\section{Verlag Graswurzelrevolution}

\section{www.graswurzel.net}

\section{Helmut Thielen \\ DIE WÜSTE LEBT \\ Jenseits von Kapital und Staat}

»Thielen kommt zu dem Schluß: Die Marktwirtschaft ist keine Zivilgesellschaft, sie ist Gewalt. Sie ist nicht (mehr) reformierbar. Dieses Buch ist ein flammendes Plädoyer für Gerechtigkeit, gegen Unterdrückung." Neues Deutschland

363 Seiten | 20,50€ | ISBN 3-9806353-3-3

\section{graswurzelrevolution}

Monatszeitung für eine gewaltfreie, herrschaftslose Gesellschaft

- Widerstand gegen Staat und Krieg

- Befreiung im Alltag

- Theoretikerlnnen des Anarchismus und der Gewaltfreiheit

- Concert for Anarchy u.v.m.

Jahresabo (10 Ausgaben) $25 €$. Probeabo* (3 Ausgaben) $5 €$ Vorkasse

* Verlängert sich ohne Kündigung zum Jahresabo. Kündigung jederzeit möglich.

\section{GWR-Vertrieb • Birkenhecker Str. 11 • D-53947 Nettersheim}

